\title{
Compact perturbations of linear differential equations in locally convex spaces
}

\author{
by
}

\author{
S. A. SHKARIN (London)
}

\begin{abstract}
Herzog and Lemmert have proven that if $E$ is a Fréchet space and $T: E \rightarrow E$ is a continuous linear operator, then solvability (in $[0,1]$ ) of the Cauchy problem $\dot{x}=T x$, $x(0)=x_{0}$ for any $x_{0} \in E$ implies solvability of the problem $\dot{x}(t)=T x(t)+f(t, x(t))$, $x(0)=x_{0}$ for any $x_{0} \in E$ and any continuous map $f:[0,1] \times E \rightarrow E$ with relatively compact image. We prove the same theorem for a large class of locally convex spaces including:
\end{abstract}

- DFS-spaces, i.e., strong duals of Fréchet-Schwartz spaces, in particular the spaces of Schwartz distributions $\mathcal{S}^{\prime}\left(\mathbb{R}^{n}\right)$, the spaces of distributions with compact support $\mathcal{E}^{\prime}(\Omega)$ and the spaces of germs of holomorphic functions $H(K)$ over an arbitrary compact set $K \subset \mathbb{C}^{n}$;

- complete LFS-spaces, i.e., complete inductive limits of sequences of Fréchet-Schwartz spaces, in particular the spaces $\mathcal{D}(\Omega)$ of test functions;

- PLS-spaces, i.e., projective limits of sequences of DFS-spaces, in particular, the spaces $\mathcal{D}^{\prime}(\Omega)$ of distibutions and $\mathcal{A}(\Omega)$ of real-analytic functions.

Here $\Omega$ is an arbitrary open domain in $\mathbb{R}^{n}$. We construct an example showing that the analogous statement for (smoothly) time-dependent linear operators is invalid already for Fréchet spaces.

1. Introduction. In this paper all linear spaces are spaces over the field $\mathbb{R}$. All locally convex topological vector spaces (LCS) are assumed to be Hausdorff. Below $\mathbb{R}$ is always the set of real numbers, $\mathbb{N}$ is the set of positive integers and $\mathbb{Z}_{+}=\mathbb{N} \cup\{0\}$.

Ordinary differential equations in abstract spaces

$$
\dot{x}(t)=f(t, x(t))
$$

( $E$ is a LCS, $f: I \times E \rightarrow E$ and $I$ is an interval of $\mathbb{R}$ ) have been intensely studied during the last decades (see e.g. [1, 4, 6-12, 17-20, 23, 29-31]). One of the reasons to study them is the fact that any partial differential equation

2000 Mathematics Subject Classification: Primary 34G20.

Key words and phrases: ordinary differential equations in locally convex spaces, existence theorem, uniqueness theorem, linear differential equations, lifting. 
can be interpreted as an ordinary differential equation in an appropriate LCS. Linear differential equations form an interesting and important subclass of ordinary differential equations in LCS (see e.g. [4, 6, 9, 10, 12, 18-20, 29, 32, $33])$. A solution of (1) is a function $x \in C^{1}(I, E)$ such that $\dot{x}(t)=f(t, x(t))$ for any $t \in I$ (we consider only strong solutions). The symbol $L(E)$ stands for the space of continuous linear operators on a LCS $E$, and $\mathcal{L}(I, E)$ is the set of continuous maps $T: I \times E \rightarrow E,(t, x) \mapsto T_{t} x$, linear with respect to $x \in E$. We say that a map $f: X \rightarrow Y$ is $M$-compact ( $X$ and $Y$ are topological spaces) if $f$ is continuous and $\overline{f(X)}$ is compact and metrizable.

Let $E$ be a LCS, $a>0, f \in C([0, a], E), x_{0} \in E$ and $g:[0, a] \times E \rightarrow E$ be $M$-compact. We consider Cauchy problems for non-perturbed and perturbed linear ordinary differential equations:

$$
\begin{aligned}
\dot{x}(t) & =T x(t), & x(0) & =x_{0}, \\
\dot{x}(t) & =T x(t)+f(t), & x(0) & =x_{0}, \\
\dot{x}(t) & =T x(t)+g(t, x(t)), & x(0) & =x_{0},
\end{aligned}
$$

where $T \in L(E)$. For the time-dependent case we consider Cauchy problems

$$
\begin{aligned}
\dot{x}(t) & =T_{t} x(t), & & x(0)=x_{0}, \\
\dot{x}(t) & =T_{t} x(t)+g(t, x(t)), & & x(0)=x_{0},
\end{aligned}
$$

where $T \in \mathcal{L}([0, a], E)$. Following $[4,32,12]$ we define

$$
\begin{aligned}
& \operatorname{ex}(E)=\left\{T \in L(E):(2) \text { is solvable in }[0, a] \text { for any } x_{0} \in E\right\}, \\
& \operatorname{ex}^{\prime}(E)=\{T \in L(E):(3) \text { is solvable in }[0, a] \\
& \text { for any } \left.\left(x_{0}, f\right) \in E \times C(\mathbb{R}, E)\right\}, \\
& \operatorname{unex}(E)=\{T \in L(E):(2) \text { is uniquely solvable in }[0, a] \\
& \text { for any } \left.x_{0} \in E\right\}, \\
& \operatorname{unex}^{\prime}(E)=\{T \in L(E):(3) \text { is uniquely solvable in }[0, a] \\
& \text { for any } \left.\left(x_{0}, f\right) \in E \times C(\mathbb{R}, E)\right\}, \\
& \overline{\operatorname{ex}}(E)=\{T \in L(E):(4) \text { is solvable in }[0, a] \\
& \left.\quad \text { for any } x_{0} \in E \text { and any } M \text {-compact map } g:[0, a] \times E \rightarrow E\right\} .
\end{aligned}
$$

The sets ex $(E), \operatorname{ex}^{\prime}(E), \operatorname{unex}(E), u_{n} x^{\prime}(E)$ and $\overline{\mathrm{ex}}(E)$ do not depend on the choice of $a>0$. Moreover, these sets do not change if one replaces $[0, a]$ in their definition by $[0, \infty)\left({ }^{1}\right)$. Obviously $\overline{\operatorname{ex}}(E) \subseteq \operatorname{ex}^{\prime}(E) \subseteq \operatorname{ex}(E)$,

$\left({ }^{1}\right)$ Indeed, if $b \in(0, \infty]$ and $T \in L(E)$ satisfies one of the conditions (7)-(11) for some $a>0$, then we can represent the interval $I=[0, b](I=[0, \infty)$ if $b=\infty)$ as a finite (or countable if $b=\infty)$ union of intervals $\left[x_{j}, x_{j+1}\right]$ such that $x_{j}<x_{j+1}$ and $x_{j+1}-x_{j} \leq a$ for any $j$. Then we can produce a solution of (2), (3) or (4) on $I$ solving the equation on $\left[x_{j}, x_{j+1}\right]$ consecutively and using the value of the solution at the right end of the previous interval as the initial data. 
unex $^{\prime}(E) \subseteq \operatorname{unex}(E) \subseteq \operatorname{ex}(E)$ and $\operatorname{unex}^{\prime}(E) \subseteq \operatorname{ex}^{\prime}(E)$. If $E$ is a Banach space then $L(E)=$ unex $^{\prime}(E)$ according to the Picard theorem. When $E$ is a Fréchet space, this equality is in general invalid. For example, $L(E) \neq \operatorname{ex}(E) \neq$ unex $(E)$ for $E=C^{\infty}[0,1]$ (see $[20,19]$ ) and $L(E)=\operatorname{ex}(E) \neq \operatorname{unex}(E)$ for $E=\mathbb{R}^{\mathbb{N}}$ (see $\left.[32,9,10]\right)$. Moreover, there exists $T \in \mathcal{L}\left(\mathbb{R}, \mathbb{R}^{\mathbb{N}}\right)$ such that $(5)$ has no solutions for any $x_{0} \in \mathbb{R}^{\mathbb{N}} \backslash\{0\}$ (see [33]). However, there exists a non-normable Fréchet space $E$ such that $L(E)=\operatorname{unex}(E)$ (see [18]). The following theorem is proved by Herzog and Lemmert [12].

Theorem HL. Let $E$ be a Fréchet space. Then $\operatorname{ex}(E)=\overline{\operatorname{ex}}(E)$.

Note that the weaker equality $\operatorname{ex}(E)=\operatorname{ex}^{\prime}(E)$ for Fréchet spaces is also proved in [29]. Let

$$
\mathcal{C P}=\{E: E \text { is a LCS and } \operatorname{ex}(E)=\overline{\operatorname{ex}}(E)\} .
$$

In this paper we prove a sufficient condition for a LCS $E$ (in terms of metric compact lifting property) to be an element of $\mathcal{C P}$. Using this condition, we prove that $\mathcal{C P}$ includes three classes of LCS $\mathcal{Y}, \mathcal{X} \cap \mathbb{U} \mathcal{F}$ and $\mathcal{X} \cap \mathbb{U}_{0} \mathbb{P} \mathbb{U}_{0}^{\mathrm{I}} \mathcal{F}$ defined below and show that $\mathcal{Y}$ contains the duals of separable metrizable locally convex spaces with the pre-compact convergence topology, $\mathcal{X} \cap \mathbb{U} \mathcal{F}$ contains the compactly regular $\left({ }^{2}\right)$ countable inductive limits of Fréchet spaces and $\mathcal{X} \cap \mathbb{U}_{0} \mathbb{P} \mathbb{U}_{0}^{\mathrm{I}} \mathcal{F}$ which contains compactly regular LCS which are countable inductive limits of countable projective limits of countable inductive limits of separable Fréchet spaces. In particular, the spaces $\mathcal{D}(\Omega)$ of infinitely differentiable functions with compact support, $\mathcal{S}^{\prime}\left(\mathbb{R}^{n}\right)$ of Schwartz distributions, $\mathcal{D}^{\prime}(\Omega)$ of generalized functions and $\mathcal{A}(\Omega)$ of real-analytic functions belong to $\mathcal{C P}$, where $\Omega$ is an open subset of $\mathbb{R}^{n}$.

Let $\mathcal{S}=\mathcal{S}(\mathbb{R})$ be the space of rapidly decreasing infinitely differentiable functions on $\mathbb{R}$ :

$$
\mathcal{S}=\left\{f \in C^{\infty}(\mathbb{R}):\|f\|_{n, k}=\sup _{x \in \mathbb{R}}\left|f^{(n)}(x)\right|\left(|x|^{k}+1\right)<\infty\right.
$$

for any $\left.n, k \in \mathbb{Z}_{+}\right\}$,

endowed with the topology defined by the seminorms $\|\cdot\|_{n, k}$. Note that $\mathcal{S}$ is a nuclear Fréchet space [21]. We construct $T \in \mathcal{L}(\mathbb{R}, \mathcal{S})$ and $y_{0} \in \mathcal{S}$ such that for any $\left(t_{0}, x_{0}\right) \in \mathbb{R} \times \mathcal{S}$, the problem

$$
\dot{x}(t)=T_{t} x(t), \quad x\left(t_{0}\right)=x_{0},
$$

is uniquely solvable in any interval containing $t_{0}$, and the equation $\dot{x}(t)=$ $T_{t} x(t)+y_{0}$ has no solutions in $[0, \varepsilon)$ for any $\varepsilon>0$. This example shows that the natural analog of Theorem HL for time-dependent linear operators on Fréchet spaces is invalid.

$\left({ }^{2}\right)$ A LCS $E$ is said to be compactly regular if it is sequentially complete and its dual $E^{\prime}$ with the convex compact convergence topology is a Schwartz space. 
We also construct a complete ultrabornological LCS which does not belong to $\mathcal{C P}$.

2. Notation and definitions. Everywhere below, $I$ is a compact interval of the real line. For a subset $A$ of a $\operatorname{LCS} E, \overline{\operatorname{aco}} A$ stands for the closure of the balanced convex hull of $A$ in $E, \mathcal{U}(E)$ is the set of open convex and balanced neighborhoods of zero in the LCS $E$, and $\mathcal{K}(E)$ is the set of convex balanced metrizable compact subsets of $E$. A set $\mathcal{B} \subseteq \mathcal{K}(E)$ is called a base of $\mathcal{K}(E)$ if any $Q \in \mathcal{K}(E)$ is contained in some $K \in \mathcal{B}$. For a convex balanced set $M \subset E, E_{M}$ stands for the linear hull of $M$, endowed with the locally convex topology $\tau=\tau(M, E)$ having the set $\{U \cap M: U \in \mathcal{U}(E)\}$ as a pre-base of neighborhoods of zero. As usual, a disk is a closed bounded convex balanced subset of a LCS $E$ (see [5]). Note that for a disk $D \subset E$, the topology $\tau(D, E)$ on $E_{D}$ is defined by the norm $p_{D}$, which is the Minkowski functional of $D$, i.e., $E_{D}$ is a normed space. For two disks $D_{1}, D_{2} \subset E$, we write $D_{1} \ll D_{2}$ if $D_{1}$ is pre-compact in the normed space $E_{D_{2}}$ and there exists $\varepsilon \in(0,1)$ such that $D_{1} \subseteq \varepsilon D_{2}$.

DeFinition 1. A LCS $E$ is said to be integrally complete if any $f \in$ $C(I, E)$ is Riemann integrable in $E$, or equivalently (see [16] for the proof), for any metrizable compact set $K \subset E$, aco $K$ is compact (and automatically metrizable [5]).

REMARK 1 . The last property is usually called the metric convex compactness property [5]. Evidently any sequentially complete LCS is integrally complete. Note that any LCS quasicomplete in the Mackey topology [13] is integrally complete [16].

Definition 2. We say that a map $T: E \rightarrow F(E$ and $F$ are LCS $)$ lifts metric compacts if for any $K \in \mathcal{K}(F)$ there exists $Q \in \mathcal{K}(E)$ such that $T(Q) \supseteq K$. We say that a pair $(E, F)$ of LCS has the metric compact lifting property if any surjective linear sequentially continuous operator $T: E \rightarrow F$ lifts metric compacts.

Definition 3. A subset $A$ of a topological space $X$ is said to be Bairemeasurable if $A$ is a symmetric difference of an open set and a Baire first category set. A topological space is called Polish if it is separable metrizable and its topology is defined by a complete metric. A topological space is called Suslin if it is a continuous image of a Polish space. A metrizable Suslin space is called analytic.

It is well known that Baire-measurable subsets of a topological space form a $\sigma$-algebra, containing the Borel $\sigma$-algebra [24]. We need several classes of LCS. As usual $\mathcal{F}$ is the class of Fréchet spaces. By $\mathcal{X}$ we denote the class of sequentially complete LCS $E$ such that for any $Q \in \mathcal{K}(E)$ there exists 
$K \in \mathcal{K}(E)$ for which $Q \ll K$. The symbol $\mathcal{Y}$ stands for the class of integrally complete LCS $E$ with $\mathcal{K}(E)$ having a countable base. If $\mathcal{A}$ is a class of LCS then by $\mathbb{U A}$ we denote the class of LCS $E$ for which there exist linear subspaces $E_{n} \subset E$ and stronger locally convex topologies $\tau_{n}$ on $E_{n}$ such that

(U0) $E=\bigcup_{n=1}^{\infty} E_{n}$ and for any $n \in \mathbb{N},\left(E_{n}, \tau_{n}\right) \in \mathcal{A}$ and $E_{n} \subseteq E_{n+1}$.

We denote by $\mathbb{P} \mathcal{A}$ the class of sequentially closed subspaces of countable products of spaces from $\mathcal{A}$. The symbol $\mathbb{U}_{0} \mathcal{A}$ stands for the class of LCS $(E, \tau)$ such that there exist linear subspaces $E_{n} \subset E$ and stronger locally convex topologies $\tau_{n}$ on $E_{n}$ satisfying (U0) and for any $n \in \mathbb{N}$, the space $\left(E_{n},\left.\tau\right|_{E_{n}}\right)$ is Suslin.

The symbols $\mathbb{U}^{\mathrm{I}} \mathcal{A}$ and $\mathbb{U}_{0}^{\mathrm{I}} \mathcal{A}$ stand for the classes of integrally complete spaces from $\mathbb{U} \mathcal{A}$ and $\mathbb{U}_{0} \mathcal{A}$ respectively.

Definition 4. Let $E$ be a LCS and $T \in \mathcal{L}(I, E)$. We denote by $\Sigma(I, T)$ the space of solutions in $I$ of the equation $\dot{x}(t)=T_{t} x(t)$, endowed with the uniform convergence topology. We say that a linear subspace $F \subseteq C(I, E)$ is an $S$-space if $F=\Sigma(I, T)$ for some $T \in \mathcal{L}(I, E)$.

Definition 5. Let $I=[a, b], E$ be a LCS, $f \in C(I \times E, E)$ and $K \in$ $\mathcal{K}(E)$. We say that equation (1) is compactly solvable over $K$ if there exists $M \in \mathcal{K}(E)$ such that

(14) for any $\left(t_{0}, x_{0}\right) \in I \times K$ there exists a solution $x:\left[t_{0}, b\right] \rightarrow M$ of the problem $\dot{x}(t)=f(t, x(t)), x\left(t_{0}\right)=x_{0}$.

We say that (1) is uniformly compactly solvable if for any $K \in \mathcal{K}(E),(1)$ is compactly solvable over $K$. We write $\mathcal{L}_{\text {ucs }}(I, E)$ for the set of $T \in \mathcal{L}(I, E)$ for which the equation $\dot{x}(t)=T_{t} x(t)$ is uniformly compactly solvable.

\section{Main results}

Proposition 1. Let $a>0, I=[0, a], E$ be an integrally complete $L C S$ and $T \in \mathcal{L}_{\text {ucs }}(I, E)$. Then for any $M$-compact map $g: I \times E \rightarrow E$ and any $x_{0} \in E$, problem (6) is solvable in $I$.

THEOREM 1. Let $E$ be an integrally complete $L C S, a>0, I=[0, a]$ and $T \in L(E)$. Suppose that

$$
\text { the operator } \mathbb{T}: \Sigma(I, T) \rightarrow E, \mathbb{T} x=x(0) \text { lifts metric compacts. }
$$

Then $T \in \overline{\mathrm{ex}}(E)$ (in particular $T \in \mathrm{ex}^{\prime}(E)$ ).

TheOREM 2. Let $E$ and $F$ be $L C S$ such that either $E \in \mathcal{Y}$ or $F \in \mathcal{X}$ and $E \in \mathbb{U}_{0} \mathbb{P} \mathbb{U}_{0} \mathcal{F} \cup \mathbb{U} \mathcal{F}$. Then the pair $(E, F)$ has the metric compact lifting property. 
Proposition 2. Let $E$ be a $L C S$ and $F \subset C(I, E)$ be an $S$-space. Then $E \in \mathcal{Y} \Rightarrow F \in \mathcal{Y}, E \in \mathcal{X} \Rightarrow F \in \mathcal{X}, E \in \mathbb{U}^{\mathrm{I}} \mathcal{F} \Rightarrow F \in \mathbb{U}^{\mathrm{I}} \mathcal{F}$ and $E \in$ $\mathbb{U}_{0}^{\mathrm{I}} \mathbb{P} \mathbb{U}_{0}^{\mathrm{I}} \mathcal{F} \Rightarrow F \in \mathbb{U}_{0}^{\mathrm{I}} \mathbb{P} \mathbb{U}_{0}^{\mathrm{I}} \mathcal{F}$.

Theorem 2 and Proposition 2 immediately imply

Corollary 1. Let $E \in(\mathcal{X} \cap \mathbb{U} \mathcal{F}) \cup\left(\mathcal{X} \cap \mathbb{U}_{0} \mathbb{P} \mathbb{U}_{0}^{\mathrm{I}} \mathcal{F}\right) \cup \mathcal{Y}$ and $F \subset C(I, E)$ be an $S$-space. Then the pair $(F, E)$ has the metric compact lifting property.

Theorem 1 and Corollary 1 imply

Corollary 2. $(\mathcal{X} \cap \mathbb{U} \mathcal{F}) \cup\left(\mathcal{X} \cap \mathbb{U}_{0} \mathbb{P} \mathbb{U}_{0}^{\mathrm{I}} \mathcal{F}\right) \cup \mathcal{Y} \subset \mathcal{C} \mathcal{P}$.

The following proposition describes properties of the classes $\mathcal{Y}$ and $\mathbb{U} \mathcal{F}$ and $\mathbb{U}_{0} \mathbb{P} \mathbb{U}_{0}^{\mathrm{I}} \mathcal{F}$. In particular, together with Corollary 1 it shows that natural LCS belong to $\mathcal{C P}$.

\section{PROPOSITION 3.}

(A) Let $E$ be a separable metrizable LCS, $E_{\tau}^{\prime}$ be its dual endowed with the locally convex topology $\tau$ such that $\sigma \subseteq \tau \subseteq \pi$, where $\sigma=\sigma\left(E^{\prime}, E\right)$ is the weak topology and $\pi=\pi\left(E^{\prime}, E\right)$ is the pre-compact convergence topology. Then $E_{\tau}^{\prime} \in \mathcal{Y}$.

(B) $\mathbb{U} \mathcal{F}$ contains all countable inductive limits of Fréchet spaces. All sequentially complete LFS-spaces (= inductive limits of sequences of Fréchet spaces) belong to $\mathcal{X} \cap \mathbb{U} \mathcal{F}$. In particular, DFS-spaces (= strong duals of Fréchet Schwartz spaces) belong to $\mathcal{X} \cap \mathbb{U F}$.

(C) $\mathbb{U}_{0} \mathbb{P} \mathbb{I}_{0}^{\mathrm{I}} \mathcal{F}$ contains all countable inductive limits of sequentially complete countable projective limits of integrally complete countable inductive limits of separable Fréchet spaces.

Corollary 3. $\mathcal{S}^{\prime}\left(\mathbb{R}^{n}\right) \in \mathcal{C P}$; for any open set $\Omega \subseteq \mathbb{R}^{n}$,

$$
\left\{\mathcal{D}(\Omega), \mathcal{D}^{\prime}(\Omega), \mathcal{A}(\Omega)\right\} \subset \mathcal{C P} ;
$$

and for any compact set $K \subset \mathbb{C}^{n}, H(K) \in \mathcal{C P}$, where $H(K)$ is the space of germs of holomorphic functions.

We construct two examples, whose properties are summarized in the following theorem.

TheOREM 3.

(i) There exist an infinitely Fréchet differentiable map $T \in \mathcal{L}(\mathbb{R}, \mathcal{S})$ and $y_{0} \in \mathcal{S}$ such that problem (5) is uniquely solvable in any interval

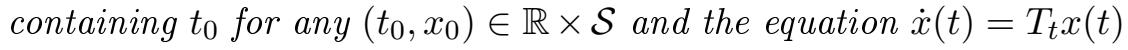
$+y_{0}$ has no solutions in $[0, \varepsilon)$ for any $\varepsilon>0$.

(ii) There exists a complete ultrabornological LCS E such that E $\notin \mathcal{C P}$. 


\section{Proofs}

\subsection{Auxiliary lemmas}

Lemma 1.1. Let $(E, \tau)$ be a LCS ( $\tau$ is the topology of $E), M \subseteq E$ be a balanced convex set, $U_{\alpha} \in \mathcal{U}(E), \alpha \in A$ be such that $\left\{U_{\alpha} \cap(2 M): \alpha \in A\right\}$ is a base of neighborhoods of zero in $\left(2 M,\left.\tau\right|_{2 M}\right)$ and $\theta$ be the (non-Hausdorff in general) locally convex topology on $E$, having the set $\left\{U_{\alpha}: \alpha \in A\right\}$ as a base of neighborhoods of zero. Then $\left.\theta\right|_{M}=\left.\tau\right|_{M}$.

Proof. Obviously $\left.\left.\theta\right|_{M} \subseteq \tau\right|_{M}$. Let $x \in M$ and $U$ be an open neighborhood of $x$ in $\left(M,\left.\tau\right|_{M}\right)$. Then there exists $W \in \mathcal{U}(E, \tau)$ such that $(x+W) \cap M \subseteq U$. Pick $\alpha \in A$ for which $U_{\alpha} \cap(2 M) \subseteq W \cap(2 M)$. Clearly $\left(x+U_{\alpha}\right) \cap M \subseteq$ $x+\left(U_{\alpha} \cap(2 M)\right)$. Therefore,

$$
\begin{aligned}
\left(x+U_{\alpha}\right) \cap M & =\left(x+\left(U_{\alpha} \cap(2 M)\right)\right) \cap M \subseteq(x+(W \cap(2 M))) \cap M \\
& \subseteq(x+W) \cap M \subseteq U .
\end{aligned}
$$

Since $\left(x+U_{\alpha}\right) \cap M$ is a neighborhood of $x$ in $\left(M,\left.\theta\right|_{M}\right)$, we obtain the inclusion $\left.\left.\theta\right|_{M} \supseteq \tau\right|_{M}$.

Lemma 1.2. Let $M$ be a paracompact subset of a LCS F, A be a convex subset of a LCS $E$ and $T: E \rightarrow F$ be a linear operator such that $T(A) \supseteq M$. Then for any $U \in \mathcal{U}(F)$ there exists a continuous map $f: M \rightarrow A$ such that $T f(y) \in y+U$ for any $y \in M$.

Proof. Evidently, $\{(y+U) \cap M: y \in M\}$ is an open cover of $M$. Since $M$ is paracompact, there exists a locally finite positive continuous partition of unity $\left\{\varrho_{\alpha}: \alpha \in \Omega\right\}$ on $M$ and a map $y: \Omega \rightarrow M$ such that $\varrho_{\alpha}(y)=0$ if $y \notin y(\alpha)+U$ for any $\alpha \in \Omega$ (see [14]). For any $\alpha \in \Omega$, let $x(\alpha) \in A$ be such that $T x(\alpha)=y(\alpha)$. Consider the map

$$
f: M \rightarrow E, \quad f(y)=\sum_{\alpha \in \Omega} \varrho_{\alpha}(y) x(\alpha) .
$$

Since $A$ is convex, we have $f(M) \subseteq A$. Local finiteness and continuity of $\varrho_{\alpha}$ imply continuity of $f$. Let $y \in M$. Since $y(\alpha) \in y+U$ when $\varrho_{\alpha}(y) \neq 0$, we obtain

$$
T f(y)=\sum_{\alpha \in \Omega} \varrho_{\alpha}(y) y(\alpha) \in \sum_{\alpha \in \Omega} \varrho_{\alpha}(y)(y+U)=y+U .
$$

Lemma 1.3. Let $E$ be a $L C S$ and $G=C(I, E)$. Then any $S$-space $F \subset G$ is closed in G. Moreover:

- $E$ is integrally complete $\Rightarrow G$ is integrally complete;

- $E$ is sequentially complete $\Rightarrow G$ is sequentially complete. 
Proof. Let $t_{0} \in I$ and $T \in \mathcal{L}(I, E)$ be such that $F=\Sigma(I, T)$. Evidently,

$$
\begin{gathered}
F=\left\{x \in G: A_{t} x=0 \text { for any } t \in I\right\}, \quad \text { where } \\
A_{t} x=x(t)-x\left(t_{0}\right)-\int_{t_{0}}^{t} T_{\tau} x(\tau) d \tau .
\end{gathered}
$$

Therefore $F$ is closed in $G$ as the intersection of the kernels of the linear continuous operators $A_{t}: G \rightarrow \bar{E}$, where $\bar{E}$ is the completion of $E$. If $E$ is sequentially complete then sequential completeness of $G$ is obvious. Let $E$ be integrally complete. It remains to prove integral completeness of $G$. Let $f:[0,1] \rightarrow G$ be a continuous map. Since $E$ is integrally complete, for any $s \in I$ there exists $\varphi(s)=\int_{0}^{1} f(t)(s) d t \in E$. Uniform continuity of the map $(t, s) \mapsto f(t)(s)$ implies continuity of $\varphi: I \rightarrow E$. Therefore there exists $\int_{0}^{1} f(t) d t=\varphi \in G$. Hence $G$ is integrally complete.

Lemma 1.4. Let $B$ be a Banach space and $A$ be a balanced convex Bairemeasurable subset of $B$. Then either $A$ is a Baire first category set, or for any $\varepsilon \in(0,1)$, the set $(1-\varepsilon) A$ is contained in the interior of $A$ in $B$.

Proof. Let $U=\{x \in B:\|x\|<1\}$. Suppose that $A$ is a Baire second category set. Since $A$ is Baire-measurable, there exist $x_{0} \in B, c>0$ and a Baire first category set $P \subset U$ such that $x_{0}+3 c(U \backslash P) \subseteq A$. Since $A$ is convex and balanced, we have $3 c(U \backslash Q) \subseteq A$, where $Q=P \cup(-P)$. Let us show that $c U \subseteq A$. Suppose that there exists $x_{1} \in c U \backslash A$. Then $\left(2 x_{1}+A\right) \cap A=\emptyset$. Therefore

$$
2 x_{1}+c U=\left(2 x_{1}+(c U \backslash A)\right) \cup\left(\left(2 x_{1}+c U\right) \backslash A\right) \subseteq\left(2 x_{1}+c Q\right) \cup 3 c Q .
$$

Since $Q$ is a Baire first category set, so is the ball $2 x_{1}+c U$, which contradicts Baire's theorem. Thus, $c U \subseteq A$. Let now $\varepsilon \in(0,1)$ and $x \in(1-\varepsilon) A$. Since $A$ is convex and balanced, $x+c \varepsilon U \subset x+\varepsilon A \subset A$. Therefore $x$ is an interior point of $A$.

Lemma 1.5. Let $E$ be a $L C S, X$ be a Suslin topological space, $K \in \mathcal{K}(E)$ and $f: X \rightarrow E$ be a sequentially continuous map. Then the set $f(X) \cap E_{K}$ is a Baire-measurable subset of the Banach space $E_{K}$.

Proof. Let $X_{n}=f^{-1}(n K)$. Then $X_{n}$ is a Suslin space as a sequentially closed subset of a Suslin space $X$. So for any $n \in \mathbb{N}$, there exists a Polish space $Y_{n}$ and a continuous surjective map $g_{n}: Y_{n} \rightarrow X_{n}$. Since $Y_{n}$ is metrizable, the map $f \circ g_{n}: Y_{n} \rightarrow E$ is continuous and therefore Borel-measurable. Since the Borel $\sigma$-algebras of subsets of $E_{K}$ with respect to the induced topology and to the Banach space topology coincide (both $\sigma$-algebras are generated by the set $\left.\left\{x+c K: x \in E_{K}, c>0\right\}\right), f \circ g_{n}$ is a Borel-measurable map from $Y_{n}$ to the Banach space $E_{K}$. According to Luzin's theorem [14], the image of a Polish space under a Borel-measurable map (taking values in a 
metric space $)$ is analytic. Hence $f\left(X_{n}\right)=\left(f \circ g_{n}\right)\left(Y_{n}\right)$ is analytic. Since any analytic subset of a metric space is Baire-measurable [14], we see that $f\left(X_{n}\right)$ is Baire-measurable in $E_{K}$. Since $f(X) \cap E_{K}$ is the union of the $f\left(X_{n}\right)$, we obtain Baire-measurability of $f(X) \cap E_{K}$ in $E_{K}$.

LEMMA 1.6.

(i) Let $E$ be a Fréchet space and $Q \in \mathcal{K}(E)$. Then there exists $K \in \mathcal{K}(E)$ such that $Q \ll K$.

(ii) Let $E$ be a $L C S, K_{1}, K_{3} \in \mathcal{K}(E)$ and $K_{1} \ll K_{3}$. Then there exists $K_{2} \in \mathcal{K}(E)$ such that $K_{1} \ll K_{2} \ll K_{3}$.

Proof. (i) is proved in [21]. For a simpler proof see Lemma 3 of [28].

(ii) Assertion (i) implies the existence of $Q \in \mathcal{K}\left(E_{K_{3}}\right)$ for which $K_{1} \ll Q$. Since $K_{1} \ll K_{3}$, there exists $q \in(0,1)$ such that $K_{1} \subseteq q^{2} K_{3}$. Clearly, $K_{2}=Q \cap q K_{3}$ satisfies the required conditions.

Lemma 1.7. Let $E$ and $F$ be LCS, $T: E \rightarrow F$ be a linear operator, $K, Q \in \mathcal{K}(F), Q \ll K$, and $U, M$ be convex balanced Suslin subsets of $E$ such that $U \subseteq M, U$ is absorbing in the linear hull of $M$, the restriction $\left.T\right|_{M}$ is sequentially continuous and $T(M) \supseteq K$. Then there exist $\varepsilon>0$ and $L \in \mathcal{K}(E)$ such that $L+\varepsilon U \subseteq M$, the linear hull of $L$ is finite-dimensional and $T(L+\varepsilon U) \supseteq Q$.

Proof. Let $\varepsilon \in(0,1)$ be such that $Q \subseteq(1-\varepsilon) K$. Since $U$ is Suslin and $\left.T\right|_{U}$ is sequentially continuous, Lemma 1.5 implies that $T(U)$ is Baire-measurable in the Banach space $F_{K}$. Since $T(U)$ is absorbing in $F_{K}$, Lemma 1.4 implies the existence of $c>0$ such that $c K \subset T(U)$. Since $Q$ is compact in $F_{K}$ and $Q \subseteq(1-\varepsilon) K$, and $T(M) \supseteq K$, there exist $x_{1}, \ldots, x_{n} \in M$ such that

$$
Q \subseteq \bigcup_{j=1}^{n}(1-\varepsilon) T x_{j}+\varepsilon T(U) \subset T(L+\varepsilon U),
$$

where $L=(1-\varepsilon) \overline{\operatorname{aco}}\left\{x_{1}, \ldots, x_{n}\right\}$. Clearly $L$ is compact and has finitedimensional linear hull. Since $L \subseteq(1-\varepsilon) M$ and $U \subseteq M$, we find that $L+\varepsilon U \subseteq M$.

Lemma 1.8. Let $(E, \theta)$ be a LCS and $M \subset E$ be a complete convex balanced metrizable set. Then $\left(E_{M}, \tau(M, E)\right)$ is a Fréchet space (see Section 2 for definitions).

Proof. Clearly $\tau=\tau(M, E)$ is stronger than $\left.\theta\right|_{E_{M}}$. Pick $U_{n} \in \mathcal{U}(E)$ such that the set $\left\{W_{n}=U_{n} \cap M: n \in \mathbb{N}\right\}$ is a base of $\tau$-neighborhoods of zero in $M$. Since for any $U \in \mathcal{U}(E)$, there exists $n \in \mathbb{N}$ such that $U \cap M \supset W_{n}$, we see that $\left\{W_{n}: n \in \mathbb{N}\right\}$ is a pre-base of $\tau$-neighborhoods of zero in $E_{M}$. Therefore $\left(E_{M}, \tau\right)$ is metrizable. It remains to prove completeness of $\left(E_{M}, \tau\right)$. Let $x_{n}$ be a $\tau$-Cauchy sequence in $E_{M}$. Since $M$ is a $\tau$-neighborhood of zero, 
there exists $c>0$ such that $x_{n} \in c M$ for any $n \in \mathbb{N}$. Since $x_{n}$ is a $\theta$-Cauchy sequence and $c M$ is complete in $(E, \theta)$, we find that $x_{n}$ is $\theta$-convergent to $x \in c M$. We have to show that $x_{n}$ is $\tau$-convergent to $x$. According to the definition of $\tau$, to this end it suffices to verify that $p_{M}\left(x_{n}-x\right) \rightarrow 0$, where $p_{M}$ is the Minkowski functional of $M$. Suppose the contrary. Then there exists $\varepsilon>0$ and an infinite set $A \subset \mathbb{N}$ such that $x_{n}-x \notin \varepsilon M$ for all $n \in A$. Since $x_{n}$ is a $\tau$-Cauchy sequence, there exists an infinite set $B \subset A$ such that $x_{n}-x_{m} \in \varepsilon M$ for all $m, n \in B$. Fixing $n \in B$, passing to the limit as $m \rightarrow \infty$ and using $\theta$-completeness of $M$, we deduce that $x_{n}-x \in \varepsilon M$, which is a contradiction.

\subsection{Proofs of Proposition 1 and Theorem 1}

Definition 6. Let $E$ be a LCS, $I=[a, b], J=\left\{(t, s) \in I^{2}: t \geq s\right\}$, $J_{b}=J \backslash\{(b, b)\}, f \in C(I \times E, E), A, B \subseteq E$ and $U \in \mathcal{U}(E)$. A map $S: J \times A \rightarrow B,(t, s, x) \mapsto S_{t}^{s} x$, is called a continuous approximate system of solutions (CASS $(A, B, U))$ of (1) if

(A1) $\quad S$ is continuous on $J \times A$ and differentiable with respect to $t$ on $J_{b} \times A$;

(A2) the derivative $\frac{\partial}{\partial t} S_{t}^{s} x$ admits a continuous extension to $J \times A$;

$$
\frac{\partial}{\partial t} S_{t}^{s} x-f\left(t, S_{t}^{s} x\right) \in U \text { for any }(t, s, x) \in J_{b} \times A ;
$$

$S_{t}^{t} x-x \in U$ for any $(t, x) \in I \times A$.

Definition 7. Let $E$ be a LCS, $f \in C(I \times E, E)$ and $K \in \mathcal{K}(E)$. Equation (1) is called $A C C$-solvable over $K$ if there exists $Q \in \mathcal{K}(E)$ such that for any $U \in \mathcal{U}(E)$ there exists a $\operatorname{CASS}(K, Q, U)$ of (1). Equation (1) is called uniformly $A C C$-solvable if for any $K \in \mathcal{K}(E)$, this equation is ACCsolvable over $K$.

LEMma 2.1. Let $E$ be an integrally complete $L C S, a>0, I=[0, a]$, $T \in \mathcal{L}(I, E), g \in C(I \times E, E), x_{0} \in E$ and $K \in \mathcal{K}(E)$ be such that $x_{0} \in K$, $g(t, x) \in K, T_{t} g(t, x) \in K$ for any $(t, x) \in I \times E$ and the equation $\dot{x}(t)=$ $T_{t} x(t)$ is ACC-solvable over $K$. Then (6) is solvable in $I$.

Proof. Without loss of generality, we can assume that $a \leq 1$. According to Definition 7 there exists $Q \in \mathcal{K}(E)$ such that $K \subseteq Q$ and for any $U \in \mathcal{U}(E)$ there exists a $\operatorname{CASS}(K, Q, U)$ of the equation $\dot{x}(t)=T_{t} x(t)$. Using integral completeness of $E$, we can choose $N \in \mathcal{K}(E)$ such that $Q \subseteq N$ and $T_{t} x \in N$ for any $(t, x) \in I \times Q$. Lemma 1.1 implies existence of $U_{n} \in \mathcal{U}(E)$ such that $2 U_{n+1} \subseteq U_{n}$ for all $n \in \mathbb{N}$ and $\left.\theta\right|_{N}=\left.\tau\right|_{N}$, where $\theta$ is the locally convex topology having the set $\left\{U_{n}: n \in \mathbb{N}\right\}$ as a base of neighborhoods of zero. We denote by ${ }^{n} S$ a $\operatorname{CASS}\left(K, Q, U_{n}\right)$ of the equation $\dot{x}(t)=T_{t} x(t)$. Let

$$
M=\{x \in C(I, E): x(t) \in 3 Q \text { and } x(t)-x(s) \in 4|t-s| Q
$$

for any $t, s \in I\}$. 
According to the Arzelà-Ascoli theorem we have $M \in \mathcal{K}(C(I, E))$. Let $\Phi_{n}: C(I, E) \rightarrow C(I, E)$ be defined by the formula

$$
\Phi_{n}(x)(t)=\int_{0}^{t} g(\tau, x(\tau)) d \tau+\int_{0}^{t} S_{t}^{\tau} T_{\tau} \int_{0}^{\tau} g(s, x(s)) d s d \tau+{ }^{n} S_{t}^{0} x_{0} .
$$

Clearly $\Phi_{n}$ is well defined and continuous. Moreover, for any $x \in C(I, E)$,

$$
\Phi_{n}(x)(t) \in K+Q+Q \subseteq 3 Q .
$$

Differentiating (17), we see that $\Phi_{n}(x) \in C^{1}(I, E)$ for any $x \in C(I, E)$ and

$$
\begin{aligned}
\frac{d}{d t} \Phi_{n}(x)(t)= & g(t, x(t))+{ }^{n} S_{t}^{t} T_{t} \int_{0}^{t} g(s, x(s)) d s \\
& +\int_{0}^{t} \frac{\partial}{\partial t}{ }^{n} S_{t}^{\tau} T_{\tau} \int_{0}^{\tau} g(s, x(s)) d s d \tau+\frac{\partial}{\partial t}{ }^{n} S_{t}^{0} x_{0} .
\end{aligned}
$$

Using (A1), (A2), (19) and the definition of $Q$, we have

$$
\frac{d}{d t} \Phi_{n}(x)(t) \in K+Q+Q+Q \subseteq 4 Q .
$$

Formulas (16), (18) and (20) imply that $\Phi_{n}(M) \subseteq M$. According to the Tikhonov fixed point theorem (see, e.g., [25]) for any $n \in \mathbb{N}$ there exists a solution $x_{n} \in M$ of the equation $\Phi_{n}(x)=x$. Since $M$ is compact and metrizable, the sequence $x_{n}$ has a subsequence $x_{n_{k}}$ uniformly converging to $x \in M$. It remains to show that $x$ is a solution of (6).

From (A4) and (17) it follows that $x_{n}(0)=\Phi_{n}\left(x_{n}\right)(0)={ }^{n} S_{0}^{0} x_{0} \in x_{0}+U_{n}$. Therefore $x_{n}(0) \rightarrow x_{0}$ with respect to $\theta$. Since $\theta$ induces the initial topology on $3 Q$ and $x_{n}(0) \in 3 Q$, we find that $x_{n}(0) \rightarrow x_{0}$. Hence $x(0)=x_{0}$. Applying (19) to $x_{n}$ and using (A3) and (A4), we see that

$$
\begin{aligned}
\dot{x}_{n}(t) \in & g\left(t, x_{n}(t)\right)+T_{t} \int_{0}^{t} g\left(s, x_{n}(s)\right) d s \\
& \quad+T_{t} \int_{0}^{t} S_{t}^{\tau} T_{\tau} \int_{0}^{\tau} g\left(s, x_{n}(s)\right) d s d \tau+T_{t}^{n} S_{t}^{0} x_{0}+3 U_{n} \\
= & g\left(t, x_{n}(t)\right)+T_{t} \Phi_{n}\left(x_{n}\right)(t)+3 U_{n}=g\left(t, x_{n}(t)\right)+T_{t} x_{n}(t)+3 U_{n} .
\end{aligned}
$$

According to (18) and (20), $\dot{x}_{n}(t)=\frac{d}{d t} \Phi_{n}\left(x_{n}\right)(t) \in 4 Q, g\left(t, x_{n}(t)\right) \in K$ and $T_{t} x_{n}(t)=T_{t} \Phi_{n}\left(x_{n}\right)(t) \in 3 N$. Therefore $\dot{x}_{n}(t)-g\left(t, x_{n}(t)\right)-T_{t} x_{n}(t) \in$ $8 N \cap 3 U_{n}$. Since $\theta$ induces the initial topology on $8 N$, it follows that $\dot{x}_{n_{k}}(t)$ uniformly converges to $T_{t} x(t)+g(t, x(t))$. Hence, $x \in C^{1}(I, E)$ and $\dot{x}(t)=$ $T_{t} x(t)+g(t, x(t))$.

Corollary 2.1. Let $E$ be an integrally complete $L C S, a>0, I=[0, a]$ and $T \in \mathcal{L}(I, E)$ be such that the equation $\dot{x}(t)=T_{t} x(t)$ is uniformly $A C C$ - 
solvable. Then for any $M$-compact map $g: I \times E \rightarrow E$ and any $x_{0} \in E$ the problem (6) is solvable in $I$.

LEMMA 2.2. Let $E$ be an integrally complete $L C S, a>0, I=[0, a]$, $K \in \mathcal{K}(E)$ and $T \in \mathcal{L}(I, E)$ be such that the equation $\dot{x}(t)=T_{t} x(t)$ is compactly solvable over $K$. Then $\dot{x}(t)=T_{t} x(t)$ is $A C C$-solvable over $K$.

Proof. According to compact solvability of the equation $\dot{x}(t)=T_{t} x(t)$ over $K$, there exists $M \in \mathcal{K}(E)$ such that (14) is satisfied. Since $E$ is integrally complete, there exists $Q \in \mathcal{K}(E)$ for which $M \subseteq Q$ and $\left\{T_{t} x:(t, x) \in\right.$ $I \times M\} \subseteq Q$. Let $U \in \mathcal{U}(E)$ and let $n \in \mathbb{N}$ be such that $\frac{4 a}{n} Q \subset U$. Define $h=a / n$ and let $j \in\{0,1, \ldots, n-1\}, F_{j}=\Sigma([j h, a], T)$. Consider the operator $\mathbb{T}_{j}: F_{j} \rightarrow E, \mathbb{T}_{j} x=x(j h)$. Formula (14) implies that $\mathbb{T}_{j}\left(M_{j}\right) \supset K$, where $M_{j}=\left\{x \in F_{j}: x([j h, a]) \subset M\right\}$. According to Lemma 1.2 there exist continuous maps $f_{j}: K \rightarrow M_{j}$ for which $\mathbb{T}_{j} f(x)-x \in U / 2$ for any $x \in K$. Let $J=\left\{(t, s) \in I^{2}: t \geq s\right\}$ and $S: J \times K \rightarrow M$ be the map defined by

$$
S_{t}^{s} x=\left\{\begin{array}{l}
f_{0}(x)(t) \\
q f_{j-2}(x)(t)+(1-q) f_{j-1}(x)(t) \\
\text { if } s=(j-q) h, 0 \leq q<1,2 \leq j \leq n .
\end{array}\right.
$$

It suffices to verify that $S$ is a $\operatorname{CASS}(K, Q, U)$ of the equation $\dot{x}(t)=$ $T_{t} x(t)$. Continuity of $S$ follows from continuity of $f_{j}$. Since for any $s$ and $x$, the map $t \mapsto S_{t}^{s} x$ is a solution of the equation $\dot{x}(t)=T_{t} x(t)$, we see that conditions (A1)-(A3) with $A=K$ and $B=Q$ are satisfied. It remains to verify (A4). Let $t \in I$ and $x \in K$. If $t>h$, we have $t=(j-q) h$ with $0 \leq q<1$ and $2 \leq j \leq n$. Therefore $S_{t}^{t} x-x=q\left(f_{j-2}(x)(t)-x\right)+$ $(1-q)\left(f_{j-1}(x)(t)-x\right)$. Since $\frac{d}{d t} f_{k}(x)(t) \in Q$ for any $(x, t) \in K \times[k h, a]$, we find that $f_{k}(t)-f_{k}(k h) \in|t-k h| Q$. The inclusion $f_{k}(k h)-x \in U / 2$ implies $S_{t}^{t} x-x \in U / 2+[q(t-(j-2) h)+(1-q)(t-(j-1) h)] Q \subseteq U / 2+2 h Q \subseteq U$. If $t \leq h$, then $S_{t}^{t} x-x=f_{0}(x)(t)-x=\left(f_{0}(x)(t)-f_{0}(x)(0)\right)+\left(f_{0}(x)(0)-x\right) \in$ $h Q+U / 2 \subseteq U$. Thus, in any case $S_{t}^{t} x-x \in U$, which is (A4).

LEMMA 2.3. Let $E$ be an integrally complete $L C S, a>0, I=[0, a]$, $K \in \mathcal{K}(E), T \in L(E), F=\Sigma(I, T)$ and $\mathbb{T}: F \rightarrow E, \mathbb{T} x=x(0)$. Suppose also that there exists $Q \in \mathcal{K}(F)$ such that $\mathbb{T}(Q) \supseteq K$. Then the equation $\dot{x}(t)=T x(t)$ is ACC-solvable over $K$.

Proof. Let $U \in \mathcal{U}(E)$ and $M=\overline{\operatorname{aco}}(\{x(t):(t, x) \in I \times Q\} \cup\{T x(t)$ : $(t, x) \in I \times Q\})$. Since $E$ is integrally complete we have $M \in \mathcal{K}(E)$. According to Lemma 1.2 there exists a continuous map $f: K \rightarrow M$ such that $\mathbb{T} f(x)-$ $x \in U$ for any $x \in K$. Let $S: J \times K \rightarrow M, S_{t}^{s} x=f(x)(t-s)$, where $J=\left\{(t, s) \in I^{2}: t \geq s\right\}$. It remains to prove that $S$ is a $\operatorname{CASS}(K, M, U)$ of the equation $\dot{x}(t)=T_{t} x(t)$. Continuity of $S$ follows from continuity of $f$. 
Since $f$ takes values in the space of solutions of the equation $\dot{x}=T x$, we have $\frac{\partial}{\partial t} S_{t}^{s} x=T f(x)(t-s) \in M$. This proves (A1)-(A3). Let $(t, x) \in I \times K$. Then $S_{t}^{t} x=f(x)(0)=\mathbb{T} f(x) \in x+U$, which is (A4).

Corollary 2.2. Let $E$ be an integrally complete $L C S, a>0, I=[0, a]$, $K \in \mathcal{K}(E)$ and $T \in \operatorname{ex}(E)$ be an operator satisfying (15). Then the equation $\dot{x}(t)=T x(t)$ is uniformly ACC-solvable in $I$.

Corollary 2.1 and Lemma 2.2 imply Proposition 1. Theorem 1 follows from Corollaries 2.1 and 2.2.

\subsection{Proof of Theorem 2}

LEMma 3.1. Let $\mathcal{G}$ be the class of LCS E such that for any LCS F, any $Q, K \in \mathcal{K}(F)$ and any sequentially continuous linear operator $T: E \rightarrow F$ such that $Q \ll K$ and $T(E) \supseteq K$ there exists $N \in \mathcal{K}(E)$ for which $T(N)$ $\supseteq Q$. Then $\mathbb{U}_{0} \mathcal{G} \subseteq \mathcal{G}$.

Proof. Let $(E, \tau) \in \mathbb{U}_{0} \mathcal{G},\left(E_{n}, \tau_{n}\right)$ be spaces satisfying (U0) and (U1), $F$ be a LCS, $Q, K \in \mathcal{K}(F), Q \ll K$ and $T: E \rightarrow F$ be a sequentially continuous linear operator such that $T(E) \supseteq K$. We have to verify the existence of $N \in \mathcal{K}(E)$ for which $T(N) \supseteq Q$. Since $\left(E_{n},\left.\tau\right|_{E_{n}}\right)$ is Suslin, Lemma 1.5 implies that $A_{n}=T\left(E_{n}\right) \cap F_{K}$ is a Baire-measurable subset of the Banach space $F_{K}$. According to Lemma 1.4, there exists $n \in \mathbb{N}$ for which $K \subset F_{K} \subset T\left(E_{n}\right)$. Since $E_{n} \in \mathcal{G}$, there exists $N \in \mathcal{K}\left(E_{n}\right)$ such that $T(N) \supseteq Q$. Since the topology $\tau_{n}$ of $E_{n}$ is stronger than $\left.\tau\right|_{E_{n}}$, we conclude that $N \in \mathcal{K}(E)$.

Lemma 3.2. Let $\mathcal{G}$ be the class of LCS defined in Lemma 3.1. Then $\mathbb{P U} U_{0} \mathcal{F} \subset \mathcal{G}$.

Proof. Let $E \in \mathbb{P U}_{0} \mathcal{F}, F$ be a LCS, $Q, K \in \mathcal{K}(F)$ be such that $Q \ll K$, and let $T: E \rightarrow F$ be a sequentially continuous linear operator such that $T(E) \supseteq K$. We have to prove the existence of $N \in \mathcal{K}(E)$ for which $T(N)$ $\supseteq Q$. According to Lemma 1.6 there exist $K_{j} \in \mathcal{K}(E)$ such that $K_{0}=K$ and $Q \ll K_{n+1} \ll K_{n}$ for any $n \in \mathbb{Z}_{+}$. Since $E \in \mathbb{P U}_{0} \mathcal{F}$ we find that $E$ is a sequentially closed linear subspace of the product of $E_{n} \in \mathbb{U}_{0} \mathcal{F}$. For any $n \in \mathbb{N}$, let $\left(E_{k}^{n}, \tau_{k}^{n}\right)$ be spaces satisfying conditions (U0) and (U1) as subspaces of $E_{n}$. Define

$$
G=\prod_{k=1}^{\infty} E_{k}, \quad G_{n}=\prod_{k=1}^{n} E_{k}
$$

and let

$$
\pi_{n}: G \rightarrow E_{n} \quad \text { and } \quad \Pi_{n}: G \rightarrow G_{n}
$$

be the natural projections. For any $n, k \in \mathbb{N}$, pick a base of neighborhoods of zero $\left\{U_{j}^{n, k}: j \in \mathbb{N}\right\}$ in $\left(E_{k}^{n}, \tau_{k}^{n}\right)$ consisting of closed balanced convex sets and 
such that $2 U_{j+1}^{n, k} \subseteq U_{j}^{n, k}$ for all $j, n, k$. Let $M_{0}=V_{0}=E$. We shall construct inductively $k_{n} \in \mathbb{N}$ and subsets $M_{n}, V_{n}$ of $E$ such that for any $n \in \mathbb{N}$,

$M_{n}=\left\{x \in E: \Pi_{n} x \in A_{n}\right\}$ and $V_{n}=\left\{x \in E: \Pi_{n} x \in B_{n}\right\}$, where $A_{n}, B_{n}$ are closed subsets of $P_{n}=\prod_{j=1}^{n} E_{k_{j}}^{j}$ (any $E_{k_{j}}^{j}$ is endowed with the topology $\left.\tau_{k_{j}}^{j}\right)$ and $B_{n} \subset\left(\prod_{j=1}^{n} U_{n}^{j, k_{j}}\right) \cap A_{n}$;

(L3) there exists $N_{n} \in \mathcal{K}(E)$ with finite-dimensional linear hull such that $M_{n}=N_{n}+V_{n}$.

Let $n \in \mathbb{N}$. Suppose that $k_{j}, M_{j}$ and $V_{j}$ satisfying (L1)-(L3) for $j<n$ (and, of course, the corresponding $A_{j}, B_{j}$ and $P_{j}$ ) are already constructed. Let

$$
W_{k}= \begin{cases}\left\{x \in E: \Pi_{n-1} x \in A_{n-1}, \pi_{n} x \in E_{k}^{n}\right\} & \text { if } k>1 \\ \left\{x \in E: \pi_{1} x \in E_{k}^{1}\right\} & \text { if } k=1 .\end{cases}
$$

Since the class of Suslin spaces is closed with respect to countable unions, countable products and sequentially closed subspaces, we deduce (according to (U1) for $E_{k}^{j}$ ) that $W_{k}$ is Suslin. Clearly, $M_{n-1}$ is the union of the $W_{k}$. Therefore, according to Lemma 1.5, $T\left(W_{k}\right) \cap F_{K_{2 n-2}}$ is an increasing sequence of Baire-measurable convex balanced subsets of the Banach space $F_{K_{2 n-2}}$, whose union contains $K_{2 n-2}$. Lemma 1.4 implies that the open unit ball $D=(0,1) \cdot K_{2 n-2}$ of $F_{K_{2 n-2}}$ is contained in the union of the interiors of $T\left(W_{k}\right) \cap F_{K_{2 n-2}}$ in $F_{K_{2 n-2}}$. Since $K_{2 n-1}$ is a compact subset of the ball $D$, there exists $k_{n} \in \mathbb{N}$ such that $K_{2 n-1} \subset T\left(W_{k_{n}}\right)$. Let

$$
B_{n}^{\prime}=\left(\prod_{j=1}^{n} U_{n}^{j, k_{j}}\right) \cap\left(B_{n-1} \times E_{k_{n}}^{n}\right), \quad V_{n}^{\prime}=\left\{x \in E: \Pi_{n} x \in B_{n}^{\prime}\right\} .
$$

Applying Lemma 1.7 to $M=W_{k_{n}}, U=V_{n}^{\prime}, Q=K_{2 n}$ and $K=K_{2 n-1}$, we see that there exist $N_{n} \in \mathcal{K}(E)$ and $\varepsilon \in(0,1)$ such that $N_{n}$ has finitedimensional linear hull, $N_{n}+\varepsilon V_{n}^{\prime} \subset W_{k_{n}}$ and $T\left(N_{n}+\varepsilon V_{n}^{\prime}\right) \supset K_{2 n}$. Let now

$$
\begin{array}{lrl}
B_{n} & =\varepsilon B_{n}^{\prime}, & V_{n}=\varepsilon V_{n}^{\prime}=\left\{x \in E: \Pi_{n} x \in B_{n}\right\}, \\
A_{n}=B_{n}+\varepsilon \Pi_{n}\left(N_{n}\right), & M_{n}=N_{n}+\varepsilon V_{n}^{\prime}=\left\{x \in E: \Pi_{n} x \in A_{n}\right\} .
\end{array}
$$

Conditions (L1)-(L3) for $k_{n}, N_{n}, V_{n}$ and $M_{n}$ follow from the construction. Let

$$
N=\bigcap_{n=1}^{\infty} M_{n}, \quad P=\prod_{n=1}^{\infty} E_{k_{n}}^{n},
$$

where $P$ is endowed with the topology of the product of $\left(E_{k_{n}}^{n}, \tau_{k_{n}}^{n}\right)$. Clearly $N \subset P \cap E$ and $N$ is convex and balanced. It suffices to prove that $N \in \mathcal{K}(E)$ and $T(N) \supseteq Q$. 
The inclusion $N \in \mathcal{K}(E)$ will be proved if we show that $N$ is a compact subset of the Polish space $P$ (the topology of $P \cap E$ induced from $P$ is stronger than the topology induced from $E$ ). According to (L3), for any $n \in \mathbb{N}, N$ is contained in the union of a finite number of shifts of $2 V_{n}$. Therefore, (L1) implies pre-compactness of $N$ in $P$. Since $P$ is a Polish space it remains to show that $N$ is sequentially closed in $P$. Let $x_{k} \in N$ be a sequence converging to $x \in P$. Since $E \cap P$ is sequentially closed in $P$, we see that $x \in E$. Let $n \in \mathbb{N}$. Since $A_{n}$ is closed in $P_{n}$, we find that $\Pi_{n}(x)=\lim \Pi_{n}\left(x_{k}\right) \in A_{n}$ and therefore $x \in M_{n}$. Hence, $x \in N$. Thus, $N \in \mathcal{K}(E)$.

Let $u \in Q$. According to (L2), for any $n \in \mathbb{N}$, there exists $y_{n} \in M_{n}$ such that $T y_{n}=u$. Since $y_{m} \in M_{n}$ for any $m \geq n$, from (L3) it follows that for any $m \geq n$, there exist $x_{m}^{n} \in N_{n}$ and $u_{m}^{n} \in V_{n}$ such that $x_{m}^{n}+u_{m}^{n}=$ $y_{m}$. Using standard diagonal procedure, we can choose a strictly increasing sequence $m_{j} \in \mathbb{N}$ such that $x_{m_{j}}^{n}$ is converging to $x^{n} \in N_{n}$ for any $n \in \mathbb{N}$. Since $y_{m_{j}}=x_{m_{j}}^{n}+u_{m_{j}}^{n} \in x^{n}+2 V_{n}$ for sufficiently large $j$ 's, from (L1) it follows that for any $n \in \mathbb{N}, \Pi_{n} y_{m_{j}}$ is a Cauchy sequence in $P_{n}$ and therefore $\Pi_{n} y_{m_{j}}$ converges to $z_{n} \in A_{n}$ with respect to the topology of $P_{n}$. Since $\tau_{k}^{n}$ is stronger than the topology induced from $E_{n}$, we see from (L1) that for any $U \in \mathcal{U}(E)$, there exists $n \in \mathbb{N}$ such that $V_{n} \subseteq U$. Therefore $y_{m_{j}}$ is a Cauchy sequence in $E$. Since $E$ is sequentially complete, $y_{m_{j}} \rightarrow y \in E$. Hence $\Pi_{n} y=z_{n} \in A_{n}$ for any $n \in \mathbb{N}$. Therefore $y \in N$ and $T y=\lim T y_{m_{j}}=u$. Thus, $T(N) \supseteq Q$.

Now we can prove Theorem 2. Let $E$ and $F$ be LCS such that either $E \in \mathcal{Y}$, or $F \in \mathcal{X}$ and $E \in \mathbb{U} \mathcal{F} \cup \mathbb{U}_{0} \mathbb{P} \mathbb{U}_{0} \mathcal{F}$, and let $T: E \rightarrow F$ be a surjective sequentially continuous linear operator and $Q \in \mathcal{K}(F)$. We have to verify that there exists $K \in \mathcal{K}(E)$ such that $T(K) \supseteq Q$.

CASE 1: $E \in \mathcal{Y}$. Let $\left\{K_{n}: n \in \mathbb{N}\right\}$ be a base of $\mathcal{K}(E)$. Then $Q$ is the union of the sets $T\left(K_{n}\right) \cap Q$. Since they are closed in $Q$, Baire's theorem implies that $T\left(K_{n}\right) \cap Q$ has non-empty interior in the balanced convex set $Q$ for some $n \in \mathbb{N}$. For this $n$ we see that $T\left(K_{n}\right) \cap Q$ absorbs $Q$. Hence, there exists $c>0$ such that $T(K) \supseteq Q$, where $K=c K_{n} \in \mathcal{K}(E)$.

CASE 2: $E \in \mathbb{U} \mathcal{F}$ and $F \in \mathcal{X}$. Let $\left(E_{n}, \tau_{n}\right) \in \mathcal{F}$ be spaces satisfying (U0). Since $F \in \mathcal{X}$, there exists $N \in \mathcal{K}(F)$ such that $Q \ll N$. Clearly $A_{n}=T^{-1}(N) \cap E_{n}$ is a convex balanced and closed subset of the Fréchet space $\left(E_{n}, \tau_{n}\right)$. Let $G_{n}=\left(E_{n}\right)_{A_{n}}$ and $G=F_{N}$. According to Lemma 1.8, each $G_{n}$ is a Fréchet space and $G$ is a Banach space. The closed graph theorem [13] implies that the restrictions $\left.T\right|_{G_{n}}: G_{n} \rightarrow G$ are continuous linear operators. Since $G$ is the union of the $L_{n}=T\left(G_{n}\right)$, there exists $n \in \mathbb{N}$ for which $L_{n}$ is a Baire second category set in the Banach space $G$, and in particular, $L_{n}$ is dense in $G$. Since $T_{n}=\left.T\right|_{G_{n}}: G_{n} \rightarrow L_{n}$ is a linear continuous surjective operator from the Fréchet space $G_{n}$ to the Baire metrizable LCS $L_{n}$, the 
open mapping theorem [5] implies that the operator $T_{n}$ is open. Therefore $L_{n}$ is isomorphic to the Fréchet space $G_{n} / \operatorname{ker} T_{n}$. Hence, $L_{n}$ is complete and therefore closed in $G$. Since $L_{n}$ is dense in $G$, we find that $L_{n}=G$. Therefore $T_{n}: G_{n} \rightarrow G$ is a surjective continuous linear operator from the Fréchet space $G_{n}$ to the Banach space $G$. Michael's selection theorem [22, 3] implies the existence of a continuous right inverse map $f: G \rightarrow G_{n}$ of $T_{n}$. Let $K=\overline{\operatorname{aco}} f(Q)$ (we take the closure with respect to the topology of the Fréchet space $\left.G_{n}\right)$. Compactness of $Q$ in $G$ implies that $K \in \mathcal{K}\left(G_{n}\right)$. Clearly $T(K)=Q$. Since the topology of $G$ is stronger than the topology induced from $E$, we conclude that $K \in \mathcal{K}(E)$.

CASE 3: $E \in \mathbb{U}_{0} \mathbb{P} \mathbb{U}_{0} \mathcal{F}$ and $F \in \mathcal{X}$. Let $\mathcal{G}$ be the class of LCS defined in Lemma 3.1. It suffices to prove that $E \in \mathcal{G}$. According to Lemma 3.1, to this end it is enough to verify the inclusion $\mathbb{P U}_{0} \mathcal{F} \subseteq \mathcal{G}$, which follows from Lemma 3.2.

\subsection{Proof of Proposition 2}

LEMma 4.1. Let $\mathcal{A}$ be a class of LCS such that any sequentially closed subspace of any element of $\mathcal{A}$ belongs to $\mathcal{A}$ and $G \in \mathcal{A} \Rightarrow C(I, G) \in \mathcal{A}$. Let also $(E, \tau)$ be a LCS and $F$ be a sequentially closed linear subspace of $C(I, E)$ (endowed with the uniform convergence topology). Then $E \in \mathbb{U}_{0}^{\mathrm{I}} \mathcal{A} \Rightarrow$ $F \in \mathbb{U}_{0}^{\mathrm{I}} \mathcal{A}$ and $E \in \mathbb{P} \mathcal{A} \Rightarrow F \in \mathbb{P} \mathcal{A}$.

Proof. Let $E \in \mathbb{U}_{0}^{\mathrm{I}} \mathcal{A},\left(E_{n}, \tau_{n}\right)$ be spaces satisfying (U0) and (U1) and $F_{n}=\left\{f \in F: f(t) \in E_{n}\right.$ for any $\left.t \in I\right\}$ be endowed with the uniform convergence topology $\theta_{n}$ in $\left(E_{n}, \tau_{n}\right)$. Then $\left(F_{n}, \theta_{n}\right) \in \mathcal{A}, F_{n} \subset F_{n+1}$ and $F_{n}$ with the topology induced from $C(I, E)$ is Suslin. According to Lemma 1.3, $F$ is integrally complete as a closed subspace of the integrally complete LCS $C(I, E)$. The inclusion $F \in \mathbb{U}_{0}^{\mathrm{I}} \mathcal{A}$ will be proved if we verify that the union of the $F_{n}$ coincides with $F$. Let $f \in F$ and $K=\overline{\operatorname{aco}}(f(I))$. Integral completeness of $E$ implies that $K \in \mathcal{K}(E)$. According to Lemma 1.5, each $G_{n}=E_{n} \cap E_{K}$ is a Baire-measurable subset of the Banach space $E_{K}$. Lemma 1.4 and Baire's theorem imply the existence of $n \in \mathbb{N}$ for which $G_{n}=E_{K}$ and therefore $f(I) \subset K \subset E_{n}$. Thus, $f \in F_{n}$ and $F$ is the union of the $F_{n}$. If $E \in \mathbb{P} \mathcal{A}$ then $E$ is a sequentially closed subspace of the product of the $E_{n} \in \mathcal{A}$. According to Lemma $1.3, F$ is a closed subspace of $C(I, E)$, which is a sequentially closed subspace of the product of the $C\left(I, E_{n}\right) \in \mathcal{A}$. Therefore $F \in \mathbb{P} \mathcal{A}$.

Now we can prove Proposition 2. Let $E \in \mathcal{Y},\left\{K_{n}: n \in \mathbb{N}\right\}$ be a base of $\mathcal{K}(E)$ and $M_{n}=\left\{x \in F: x(t) \in K_{n} \forall t \in I\right\}$. According to Lemma 1.3, $F$ is integrally complete. Since $E$ is integrally complete, for any $n \in \mathbb{N}$, there exists $m \in \mathbb{N}$ such that $\left\{T_{t} x: t \in I, x \in K_{n}\right\} \subseteq K_{m}$. Hence, $\{\dot{x}(t): t \in I$, $\left.x \in M_{n}\right\} \subseteq K_{m}$. The Arzelà-Ascoli theorem implies that $M_{n} \in \mathcal{K}(F)$. Suppose now that $M \in \mathcal{K}(F)$. Since $E$ is integrally complete, there exists $n \in \mathbb{N}$ 
such that $\{x(t): x \in M, t \in I\} \subseteq K_{n}$. Hence, $M \subseteq M_{n}$. Thus, $\left\{M_{n}: n \in \mathbb{N}\right\}$ is a base of $\mathcal{K}(F)$ and therefore $F \in \mathcal{Y}$.

Let $E \in \mathcal{X}, M \in \mathcal{K}(F)$ and $T \in \mathcal{L}(I, E)$ be such that $F=\Sigma(I, T)$. According to Lemma $1.3, F$ is sequentially complete. Since $E$ is integrally complete, $Q=\overline{\operatorname{aco}}\{x(t):(t, x) \in I \times M\} \in \mathcal{K}(E)$, and since $E \in \mathcal{X}$, there exists $K \in \mathcal{K}(E)$ such that $Q \ll K$. Let $N=\{x \in F: x(t) \in K \forall t \in I\}$. Since the set $\left\{\dot{x}(t)=T_{t} x(t): x \in N, t \in I\right\}$ is compact, the Arzelà-Ascoli theorem implies compactness of $N$. Thus, $N \in \mathcal{K}(E)$. Let us verify that $M \ll N$. To this end we have to show that the topology on $M$ defined by the norm $p_{N}$ coincides with the uniform convergence topology. Let $x_{n} \in M$ be a sequence uniformly converging to zero. It suffices to prove that $p_{N}\left(x_{n}\right) \rightarrow 0$. Suppose that $p_{N}\left(x_{n}\right) \nrightarrow 0$. According to the definition of $N$, there exists a sequence $t_{n} \in I$ such that $p_{K}\left(x_{n}\left(t_{n}\right)\right) \nrightarrow 0$. Since $x_{n}\left(t_{n}\right) \in Q$ and $Q \ll K$, we have $x_{n}\left(t_{n}\right) \nrightarrow 0$ in $E$, which contradicts the uniform convergence of $x_{n}$ to 0 . Hence, $M \ll N$ and therefore $F \in \mathcal{X}$.

Let $E \in \mathbb{U}^{\mathrm{I}} \mathcal{F}$, let $\left(E_{n}, \tau_{n}\right)$ be spaces satisfying (U0) and let $F_{n}=\{f \in$ $F: f(t) \in E_{n}$ for any $\left.t \in I\right\}$ be endowed with the uniform convergence topology $\theta_{n}$ in $\left(E_{n}, \tau_{n}\right)$. Then $\left(F_{n}, \theta_{n}\right) \in \mathcal{F}$ and $F_{n} \subset F_{n+1}$. According to Lemma $1.3, F$ is integrally complete as a closed subspace of the integrally complete LCS $C(I, E)$. The inclusion $F \in \mathbb{U}^{\mathrm{I}} \mathcal{A}$ will be proved if we verify that the union of the $F_{n}$ coincides with $F$. Let $f \in F$ and $K=\overline{\operatorname{aco}}(f(I))$. Integral completeness of $E$ implies that $K \in \mathcal{K}(E)$. Since $\tau_{n}$ is stronger than $\left.\tau\right|_{E_{n}}$, we have $K \in \mathcal{K}\left(E_{n}\right) \subset \mathcal{M}\left(E_{n}\right)$. According to Lemma 1.8, $G_{n}=\left(E_{n}\right)_{K}$ is a Fréchet space (with the topology $\eta_{n}$ having the set $\left\{U \cap K: U \in \mathcal{U}\left(E_{n}, \tau_{n}\right)\right\}$ as a pre-base of neighborhoods of zero). Pick $n \in \mathbb{N}$ for which $G_{n}=E_{K} \cap E_{n}$ is a Baire second category subset of the Banach space $E_{K}$. For this $n$, the identity operator $J$ from $\left(G_{n}, \eta_{n}\right)$ to $\left(G_{n}, p_{K}\right)$ is a surjective continuous linear operator from a Fréchet space to a Baire normed LCS. The open mapping theorem [5] implies that $J$ is open and therefore $J$ is an isomorphism. Hence, $\left(G_{n}, p_{K}\right)$ is complete and therefore $G_{n}$ is closed in the Banach space $E_{K}$. On the other hand, $G_{n}$ is dense in $E_{K}$ and therefore $G_{n}=E_{K}$. Hence, $f(I) \subset K \subset E_{n}$. Thus, $f \in F_{n}$ and $F$ is the union of the $F_{n}$.

Let $E \in \mathbb{U}_{0}^{\mathrm{I}} \mathbb{P} \mathbb{U}_{0}^{\mathrm{I}} \mathcal{F}$. Clearly $\mathbb{U}_{0}^{\mathrm{I}} \mathcal{F}$ is closed under taking sequentially closed linear subspaces. Lemma 4.1 applied to $\mathcal{A}=\mathcal{F}$ shows that $G \in \mathbb{U}_{0}^{\mathrm{I}} \mathcal{F} \Rightarrow$ $C(I, G) \in \mathbb{U}_{0}^{\mathrm{I}} \mathcal{F}$. Lemma 4.1 applied to $\mathcal{A}=\mathbb{U}_{0}^{\mathrm{I}} \mathcal{F}$ implies that $G \in \mathbb{P} \mathbb{U}_{0}^{\mathrm{I}} \mathcal{F} \Rightarrow$ $C(I, G) \in \mathbb{P} \mathbb{U}_{0}^{\mathrm{I}} \mathcal{F}$. Since $\mathbb{P} \mathbb{U}_{0}^{\mathrm{I}} \mathcal{F}$ is closed under taking sequentially closed linear subspaces, applying Lemma 4.1 to $\mathcal{A}=\mathbb{P} \mathbb{U}_{0}^{\mathrm{I}} \mathcal{F}$, we obtain $F \in \mathbb{U}_{0}^{\mathrm{I}} \mathbb{P} \mathbb{U}_{0}^{\mathrm{I}} \mathcal{F}$.

4.5. Proof of Proposition 3. Statement (A) follows directly from the Banach-Dieudonné theorem [13, 21]. For $(B)$, clearly $\mathbb{U} \mathcal{F}$ contains countable inductive limits of Frechét spaces. Let $E$ be a sequentially complete LFSspace and $K \in \mathcal{K}(E)$. Then $K \in \mathcal{K}\left(E_{n}\right)$ for some step space $E_{n}$ and according 
to Lemma 1.6 there exists $Q \in \mathcal{K}\left(E_{n}\right) \subset \mathcal{K}(E)$ such that $K \ll Q$. Hence $E \in \mathcal{X}$. Part $(\mathrm{C})$ follows from the definition of the class $\mathbb{U}_{0} \mathbb{P} \mathbb{U}_{0}^{\mathrm{I}} \mathcal{F}$.

4.6. Proof of Corollary 3. Since $\mathcal{D}=\mathcal{D}(\Omega)$ is a strict inductive limit of separable Fréchet spaces and $H(K), \mathcal{S}^{\prime}\left(\mathbb{R}^{n}\right)$ are strong duals of FréchetSchwartz spaces [13], Proposition 3 implies that $\mathcal{D}(\Omega), \mathcal{S}^{\prime}\left(\mathbb{R}^{n}\right), H(K) \in \mathcal{X} \cap$ $\mathbb{U}_{0}^{\mathrm{I}} \mathcal{F} \subset \mathcal{X} \cap \mathbb{U} \mathcal{F}$. According to Corollary 2, $\mathcal{D}(\Omega), \mathcal{S}^{\prime}\left(\mathbb{R}^{n}\right), H(K) \in \mathcal{C} \mathcal{P}$. Since $\mathcal{D}^{\prime}(\Omega)$ and $\mathcal{A}(\Omega)$ are projective limits of complete inductive limits of separable Fréchet spaces, Proposition 3 implies that $\mathcal{D}^{\prime}(\Omega), \mathcal{A}(\Omega) \in \mathbb{P} \mathbb{U}_{0}^{\mathrm{I}} \mathcal{F}$ $\subset \mathbb{U}_{0} \mathbb{P} \mathbb{U}_{0}^{\mathrm{I}} \mathcal{F}$. On the other hand $\mathcal{D}^{\prime}(\Omega), \mathcal{A}(\Omega) \in \mathcal{X}\left({ }^{3}\right)$. Corollary 2 now implies that $\mathcal{A}(\Omega) \in \mathcal{C P}$ and $\mathcal{D}^{\prime}(\Omega) \in \mathcal{C P}$.

\subsection{Proof of Theorem 3}

Example 1. Let $\alpha, \varphi, \beta: \mathbb{R} \rightarrow \mathbb{R}$ be functions defined by

$$
\begin{aligned}
& \alpha(s)=e^{-s^{2}}, \quad \varphi(t)= \begin{cases}e^{-t^{-4}} & \text { if } t>0, \\
0 & \text { if } t \leq 0,\end{cases} \\
& \beta(t)= \begin{cases}1 & \text { if } t \leq 0, \\
0 & \text { if } t \geq 1, \\
e^{-(1-t)^{-2}}\left(e^{-t^{-2}}+e^{-(1-t)^{-2}}\right)^{-1} & \text { if } t \in(0,1) .\end{cases}
\end{aligned}
$$

One can easily see that $\varphi, \beta \in C^{\infty}(\mathbb{R})$ and $\alpha \in \mathcal{S}$. Consider functions $\gamma, \nu^{u}$ : $\mathbb{R}^{2} \rightarrow \mathbb{R}$

(22) $\gamma(t, s)=\left\{\begin{array}{ll}\varphi(t)+\beta\left(s-t^{-1}\right) & \text { if } t>0, \\ 1 & \text { if } t \leq 0,\end{array} \quad \nu^{u}(t, s)=\frac{\gamma(t, s)}{\gamma(u, s)} \quad(u \in \mathbb{R})\right.$.

Let us verify that $\gamma \in C^{\infty}\left(\mathbb{R}^{2}\right)$. Clearly $\gamma \in C^{\infty}((0,+\infty) \times \mathbb{R})$ and $\gamma \in C^{\infty}((-\infty, 0) \times \mathbb{R})$. Let $s_{0} \in \mathbb{R}$. Pick $\varepsilon>0$ such that $s_{0}+\varepsilon-\varepsilon^{-1}<0$. Then for any $(t, s)$ from the $\varepsilon$-neighborhood $W$ of the point $\left(0, s_{0}\right)$ in $\mathbb{R}^{2}$, we have $\beta\left(s-t^{-1}\right)=1$ and therefore $\gamma(t, s)=1+\varphi(t)$. Hence $\gamma \in C^{\infty}(W)$. Therefore $\gamma \in C^{\infty}\left(\mathbb{R}^{2}\right)$. Clearly $\gamma$ is positive. Hence $\nu^{u}, \mu \in C^{\infty}\left(\mathbb{R}^{2}\right)$, where

$$
\mu(t, s)=\frac{\partial}{\partial t} \ln \gamma(t, s)= \begin{cases}\frac{\varphi^{\prime}(t)+t^{-2} \beta^{\prime}\left(s-t^{-1}\right)}{\varphi(t)+\beta\left(s-t^{-1}\right)} & \text { if } t>0, \\ 0 & \text { if } t \leq 0 .\end{cases}
$$

For any $t, u \in \mathbb{R}$, let $T_{t}$ and $S_{t}^{u}$ be operators (acting on functions $x: \mathbb{R} \rightarrow \mathbb{R}$ ) defined by the formula

$$
T_{t} x(s)=\mu(t, s) x(s), \quad S_{t}^{u} x(s)=\nu^{u}(t, s) x(s) .
$$

$\left({ }^{3}\right)$ Any compact subset $K$ of $E \in\left\{\mathcal{D}^{\prime}(\Omega), \mathcal{A}(\Omega)\right\}$ is contained in a linear subspace $F \subset E$ carrying a stronger topology $\tau$ such that $(F, \tau)$ is a Fréchet space and $K$ is compact in $(F, \tau)$. Then it remains to apply Lemma 1.6. 
For the proof of the first part of Theorem 3 it suffices to verify the following conditions:

(1.0) $T, S^{u} \in \mathcal{L}(\mathbb{R}, \mathcal{S})$, and the maps $T, S^{u}: \mathbb{R} \times \mathcal{S} \rightarrow \mathcal{S}(u \in \mathbb{R})$ are infinitely Fréchet differentiable [2], where $S^{u}(t, x)=S_{t}^{u} x$ ( $T$ and $S$ are defined by (24));

(1.1) for any $\left(t_{0}, x_{0}\right) \in \mathbb{R} \times \mathcal{S}$, problem (13) is uniquely solvable in any interval containing $t_{0}$. The unique solution is given by $x(t)=S_{t}^{t_{0}} x_{0}$; the equation $\dot{x}(t)=T_{t} x(t)+\alpha$ has no solutions in $[0, \varepsilon]$ for any $\varepsilon>0$.

Proof. (1.0) Let $\mathcal{B}$ be the set of functions $\varrho \in C^{\infty}\left(\mathbb{R}^{2}\right)$ such that for any $n, k \in \mathbb{Z}_{+}$, there exist $m=m(\varrho, n, k), c=c(\varrho, n, k)>0$ for which

$$
\sup _{t \in \mathbb{R}}\left|\frac{\partial^{n+k} \varrho}{\partial t^{n} \partial s^{k}}(t, s)\right| \leq c(1+|s|)^{m} \quad \text { for any }(t, s) \in \mathbb{R}^{2} .
$$

According to (12) it suffices to prove that $\mu \in \mathcal{B}$ and $\nu^{u} \in \mathcal{B}$ for any $u \in \mathbb{R}$. We shift the proof of this fact to the Appendix (it is purely technical).

$(1.1)$ Let $x: \mathbb{R} \rightarrow \mathcal{S}, x(t)=S_{t}^{t_{0}} x_{0}$. Clearly $x\left(t_{0}\right)=x_{0}$. By $(23), \frac{\partial \gamma}{\partial t}(t, s)=$ $\mu(t, s) \gamma(t, s)$. Therefore

$$
\dot{x}(t)(s)=\mu(t, s) \gamma(t, s) x_{0}(s) / \gamma\left(t_{0}, s\right)=\mu(t, s) x(t)(s)=T_{t} x(t)(s) .
$$

Hence, $x$ is a solution in $\mathbb{R}$ of (13). Uniqueness of a solution of this problem follows from the fact that for any $t \in \mathbb{R}, T_{t}$ is an operator of multiplication by a function (we can "pointwise" solve the equation $\dot{x}(t)=T_{t} x(t)$ ).

(1.2) Let $\varepsilon>0$. One can easily verify that the function $\pi:(0, \varepsilon] \rightarrow \mathcal{S}$,

$$
\pi(t)=(t-\varepsilon) \alpha+\int_{t}^{\varepsilon}(\varepsilon-\tau) S_{t}^{\tau} T_{\tau} \alpha d \tau
$$

is a solution of the equation $\dot{x}(t)=T_{t} x(t)+\alpha$. Suppose that there exists a solution $\pi_{1}:[0, \varepsilon] \rightarrow \mathcal{S}$ of this equation in $[0, \varepsilon]$. Then $x=\pi-\pi_{1}$ is a solution of the homogeneous equation $\dot{x}=T_{t} x$ in $(0, \varepsilon]$. According to (1.1), $x$ admits an infinitely differentiable extension to $\mathbb{R}$. Therefore $\pi$ has a limit in 0 in the topology of $\mathcal{S}$. Hence, the limit

$$
\lim _{t \downarrow 0} f(t), \quad \text { where } f(t)=\int_{t}^{\varepsilon}(\tau-\varepsilon) S_{t}^{\tau} T_{\tau} \alpha d \tau,
$$

exists in $\mathcal{S}$. Since convergence in $\mathcal{S}$ implies uniform convergence,

$$
0=\lim _{t \downarrow 0} f(1 / t)=\lim _{t \downarrow 0}(1+\varphi(t)) e^{-t^{-2}} I(t)=\lim _{t \downarrow 0} e^{-t^{-2}} I(t),
$$

where

$$
I(t)=\int_{t}^{\varepsilon}(\tau-\varepsilon) \frac{\mu(\tau, 1 / t)}{\gamma(\tau, 1 / t)} d \tau .
$$

Integrating by parts, we see that for sufficiently small positive $t$, 
(27) $I(t)=\frac{t-\varepsilon}{1+\varphi(t)}+\int_{t}^{2 t} \frac{d \tau}{\gamma(\tau, 1 / t)}+\int_{2 t}^{\varepsilon} \frac{d \tau}{\varphi(\tau)} \geq-\varepsilon+\int_{2 t}^{4 t} e^{\tau^{-4}} d \tau \geq 2 t e^{(2 t)^{-4}}-\varepsilon$. From (26) and (27) it follows that $0=\lim _{t \downarrow 0} e^{-t^{-2}}\left(2 t e^{(2 t)^{-4}}-\varepsilon\right)$. On the other hand, this limit is obviously infinite. This contradiction proves (1.2).

ExAmple 2. As usual, $\mathcal{E}=C^{\infty}(\mathbb{R})$ with the topology of uniform convergence of all derivatives, and $\mathcal{E}^{\prime}$ is the strong dual of the nuclear Fréchet space $\mathcal{E}$, i.e., $\mathcal{E}^{\prime}$ is the space of generalized functions with compact support [21]. As usual, $\delta_{t} \in \mathcal{E}^{\prime}$ is Dirac's delta-function concentrated at the point $t \in \mathbb{R}:\left\langle\delta_{t}, h\right\rangle=h(t), h \in \mathcal{E}$. Let $G$ be the linear hull in $\mathcal{E}^{\prime}$ of the set $\left\{\delta_{0}^{(n)}: n \in \mathbb{Z}_{+}\right\}$. For any $z \in \mathbb{C}$, let $e_{z} \in \mathcal{E}, e_{z}(t)=e^{-t z}$. Consider the space (28) $E=\left\{\Phi_{\xi}: \xi \in \mathcal{E}^{\prime} \otimes \mathcal{E}^{\prime}\right\}, \quad$ where $\Phi_{\xi}\left(s_{1}, s_{2}\right)=\left(s_{1}-s_{2}\right)\left\langle e_{s_{1}} \otimes e_{s_{2}}, \xi\right\rangle$.

Clearly $E$ is a linear subspace of the space of entire functions of two variables. We endow $E$ with the strongest locally convex topology with respect to which the operators

$$
\begin{aligned}
& T_{\psi}^{1}: \mathcal{E}^{\prime} \rightarrow E, \quad T_{\psi}^{1} \varphi=\Phi_{\varphi \otimes \psi}, \quad \psi \in \mathcal{E}^{\prime}, \\
& T_{\varphi}^{2}: \mathcal{E}^{\prime} \rightarrow E, \quad T_{\varphi}^{2} \psi=\Phi_{\varphi \otimes \psi}, \quad \varphi \in G,
\end{aligned}
$$

are continuous. Let $T: E \rightarrow E$ be the operator defined by the formula $T \Phi_{\varphi \otimes \psi}=\Phi_{\varphi^{\prime} \otimes \psi}$ and $f:[0,1] \rightarrow E, f(t)=\Phi_{\delta \otimes \delta_{t}}$.

For the proof of the second part of Theorem 3 it suffices to verify the following conditions:

$E$ is a complete ultrabornological LCS, $T \in L(E)$ and $f \in C([0,1], E)$; $T \in \operatorname{unex}(E)$ and for all $\varepsilon>0$, the equation $\dot{x}(t)=T x(t)+f(t)$ has no solutions in $[0, \varepsilon]$.

Proof. (2.0) One can easily see that $E_{1}=\left\{\Phi_{\xi}: \xi \in G \otimes \mathcal{E}^{\prime}\right\} \subset E$ is isomorphic to a free locally convex sum of a countable family of copies of $\mathcal{E}^{\prime}$. Therefore $E_{1}$ is complete and hence closed in $E$. On the other hand, the quotient $E / E_{1}$ is isomorphic to the free locally convex sum of a continuum of copies of $\mathcal{E}^{\prime}$ and therefore is also complete. The three-space theorem for completeness [5] implies that $E$ is complete. Moreover, the space $E$ is ultrabornological as an inductive limit of ultrabornological LCS. Since $T T_{\psi}^{1}=T_{\psi}^{1} D$, where $D: \mathcal{E}^{\prime} \rightarrow \mathcal{E}^{\prime}$ is the operator of differentiation $D \varphi=\varphi^{\prime}$ and $T T_{\varphi}^{2}=T_{\varphi^{\prime}}^{2}$, we find that the operators $T T_{\psi}^{1}$ for $\psi \in \mathcal{E}^{\prime}$ and $T T_{\varphi}^{2}$ for $\varphi \in G$ are continuous. According to the definition of the topology of $E$ we see that $T \in L(E)$. Since the function $g:[0,1] \rightarrow \mathcal{E}^{\prime}, g(t)=\delta_{t}$, is continuous and $f(t)=T_{\delta_{0}}^{2} g(t)$, we see that $f$ is continuous.

(2.1) According to (28) and to the definition of the operator $T$ we have

$$
T \Phi\left(s_{1}, s_{2}\right)=s_{1} \Phi\left(s_{1}, s_{2}\right) \quad \text { for any } \Phi \in E \text {. }
$$


Since $T$ is the operator of multiplication by a function and the topology of $E$ is stronger than the pointwise convergence topology, the "pointwise" solution of problem (2) coincides with the conventional solution in $E$, whenever the latter exists. This proves uniqueness of the solution. One can easily verify that the "pointwise" solution of problem (2) is given by

$$
x(t)\left(s_{1}, s_{2}\right)=e^{-t s_{1}} x_{0}\left(s_{1}, s_{2}\right) .
$$

Clearly $e^{-t s_{1}} \Phi_{\varphi \otimes \psi}\left(s_{1}, s_{2}\right)=\Phi_{\varphi_{t}, \psi}\left(s_{1}, s_{2}\right)$, where $\varphi_{t}$ is a shift of the generalized function $\varphi: \varphi_{t}(u)=\varphi(u-t)$. Hence the function $x$ defined by (30) takes values in $E$. Smoothness of $x:[0,1] \rightarrow E$ follows from the definition (29) of the topology of $E$ and smoothness of the functions $t \mapsto \varphi_{t}\left(\varphi \in E^{\prime}\right)$. Therefore formula (30) defines the solution in $E$ of problem (2). Hence, $T \in \operatorname{unex}(E)$.

It remains to show that the problem $\dot{x}(t)=T x(t)+f(t), x(0)=0$, is nonsolvable in $[0, \varepsilon]$. One can verify that the "pointwise" solution of this problem is given by $x\left(s_{1}, s_{2}\right)=e^{-t s_{2}}-e^{-t s_{1}}$. Suppose that the problem is solvable in $[0, \varepsilon]$. Then for any $t \in[0, \varepsilon]$ there exists $\xi_{t} \in \mathcal{E}^{\prime} \otimes \mathcal{E}^{\prime}$ such that $\Phi_{\xi_{t}}\left(s_{1}, s_{2}\right)=$ $e^{-t s_{2}}-e^{-t s_{1}}$. Therefore the function $F_{t}\left(s_{1}, s_{2}\right)=\left(e^{-t s_{2}}-e^{-t s_{1}}\right) /\left(s_{1}-s_{2}\right)$ is the Laplace transform of $\xi_{t}$ (see definition of $\Phi_{\xi}$ ). On the other hand, $F_{t}$ for $t>0$ is the Laplace transform of the Lebesgue measure $\mu_{t}$ of the segment on the plane with ends $(0, t)$ and $(t, 0)$. Injectivity of the Laplace transform implies that $\mu_{t}$ is an element of $\mathcal{E}^{\prime} \otimes \mathcal{E}^{\prime}$, which is false.

\section{Concluding remarks}

1. It is not known whether there exist a Fréchet space $E, T \in \mathcal{L}(\mathbb{R}, E)$ and $f \in C(\mathbb{R}, E)$ such that problem (13) is solvable in $\mathbb{R}$ for any $\left(t_{0}, x_{0}\right) \in \mathbb{R} \times E$ and the equation $\dot{x}(t)=T_{t} x(t)+f(t)$ has no solutions in any interval.

2. It is not known whether the product of any two spaces from $\mathcal{C P}$ belongs to $\mathcal{C P}$. Nor do we know whether $\mathcal{C P}$ contains strong duals of Fréchet spaces.

3. Let $E$ be a non-complete normed space, admitting a bigger complete norm (for example $C[0,1]$ with the norm induced from $L_{2}[0,1]$ ). Then $0 \in$ $\operatorname{unex}(E) \backslash \operatorname{ex}^{\prime}(E)$.

4. We say that a pair $(E, F)$ of LCS has the inverse mapping property if any bijective continuous linear operator $T: E \rightarrow F$ has continuous inverse.

Proposition 4. Let $E$ be an integrally complete $L C S, T \in \operatorname{unex}(E)$, $F=\Sigma([0, a], E)$, and suppose the pair $(F, E)$ has the inverse mapping property. Then $T \in \overline{\mathrm{ex}}(E)$.

Proof. Let $\mathbb{T}: F \rightarrow E, \mathbb{T} x=x(0)$ and $J=\{(t, s) \in[0, a]: t \geq s\}$. Then $\mathbb{T}$ is continuous linear and bijective and therefore $\mathbb{T}^{-1}$ is continuous. Hence, the map $S: J \times E \rightarrow E, S_{t}^{s} x=\mathbb{T}^{-1} x(t-s)$, is continuous. So for any $K \in \mathcal{K}(E)$, there exists $Q \in \mathcal{K}(E)$ such that $S_{t}^{s} x \in Q$ and $T S_{t}^{s} x \in Q$ 
for all $(t, s, x) \in J \times K$. It remains to notice that for any $U \in \mathcal{U}(E), S$ is a $\operatorname{CASS}(K, Q, U)$ of the equation $\dot{x}=T x$ and to apply Lemma 2.1 .

5 . The following proposition was suggested by D. Vogt.

Proposition 5. Let $E \in \mathcal{X}$ and $T \in L(E)$ be such that for any $K \in$ $\mathcal{K}(E)$ there exists $Q \in \mathcal{K}(E)$ such that $K \subseteq Q$ and $Q$ absorbs $T(Q)$. Then $T \in \operatorname{unex}(E) \cap \overline{\operatorname{ex}}(E)$.

Proof. Let $x_{0} \in E$ and $g:[0, a] \times E \rightarrow E$ be an $M$-compact map. Then we can pick $K, Q \in \mathcal{K}(E)$ such that $g([0, a] \times E) \subset K, x_{0} \in K, K \ll Q$ and $Q$ absorbs $T(Q)$. So the restriction $\left.T\right|_{E_{Q}}: E_{Q} \rightarrow E_{Q}$ is a continous linear operator on the Banach space $E_{Q}$ and the restriction $\left.g\right|_{[0, a] \times E_{Q}}:[0, a] \times E_{Q} \rightarrow$ $E_{Q}$ is an $M$-compact map. Theorem HL implies solvability of (4) in $E_{Q}$ and therefore in $E$. Hence $T \in \overline{\operatorname{ex}}(E)$. Let now $x \in C^{1}([0, a], E)$ be a solution of (2) with $x_{0}=0$. Pick $K, Q \in \mathcal{K}(E)$ such that $x([0, a]) \subset K, K \ll Q$ and $Q$ absorbs $T(Q)$. Then the restriction $\left.T\right|_{E_{Q}}: E_{Q} \rightarrow E_{Q}$ is a continous linear operator on the Banach space $E_{Q}$ and $x$ is a solution of (2) with $x_{0}=0$ in $E_{Q}$. Since $L\left(E_{Q}\right)=\operatorname{unex}\left(E_{Q}\right)$, we have $x \equiv 0$. Hence $T \in \operatorname{unex}(E)$.

Acknowledgments. The author would like to thank Professors O. Smolyanov, D. Vogt and G. Herzog for their interest and comments, and the referee for useful remarks. The author appreciates the financial support of the Alexander von Humboldt Foundation and the British Engineering and Physical Research Council Grant GR/T25552/01.

Appendix: Proof of the inclusions $\mu \in \mathcal{B}$ and $\nu^{u} \in \mathcal{B}$, required in Section 4.7. In this appendix we prove that the functions $\mu$ and $\nu^{u}(u \in \mathbb{R})$ defined by (23) and (22) belong to the class $\mathcal{B}$ defined by (25). Since for any fixed $u \in \mathbb{R}$, the function $s \mapsto \gamma(u, s)$ is bounded from below by a positive constant, it suffices to prove that $\mu, \gamma \in \mathcal{B}$. We write $F \preccurlyeq G(F$ and $G$ are functions defined on the same set) if there exists $c=c(F, G)>0$ such that $|F| \leq c|G|$.

First, let us show that $\gamma \in \mathcal{B}$. Clearly the function

$$
\frac{\partial^{n+k} \gamma}{\partial t^{n} \partial s^{k}}(t, s):(0,+\infty) \times \mathbb{R} \rightarrow \mathbb{R}
$$

is a finite linear combination of $\varphi^{(i)}(t)$ and $t^{-l} \beta^{(j)}\left(s-t^{-1}\right)$, where $l, i, j \in \mathbb{Z}_{+}$ and $l \leq 2 j$. The functions $\varphi^{(i)}(t)$ and $t^{-l} \beta^{(j)}\left(s-t^{-1}\right)$ for $l=0$ are bounded. If $l>0$ then $j>0$ and according to $(21), t^{-l} \beta^{(j)}\left(s-t^{-1}\right)$ vanishes if $s-t^{-1} \notin(0,1)$. This implies that $t^{-l} \beta^{(j)}\left(s-t^{-1}\right) \preccurlyeq 1+|s|^{l}$. Hence, $\gamma \in \mathcal{B}$ (and therefore $\nu^{u} \in \mathcal{B}$ ).

It remains to show that $\mu \in \mathcal{B}$. Using de la Vallée Poussin's formula for multiple derivatives of a superposition of two functions [26] we deduce that 


$$
\frac{\partial^{n+k} \mu}{\partial t^{n} \partial s^{k}}(t, s)=\frac{\partial^{n+1+k} \ln (\gamma)}{\partial t^{n+1} \partial s^{k}}(t, s)
$$

is a linear combination with real coefficients of the following finite set of functions:

$$
\frac{1}{\gamma^{\nu}(t, s)} \prod_{i, j}\left(\frac{\partial^{i+j} \gamma}{\partial t^{i} \partial s^{j}}(t, s)\right)^{a_{i, j}},
$$

where $i, j, a_{i, j} \in \mathbb{Z}_{+}, \nu=\sum_{i, j} a_{i, j}, \sum_{i, j} i a_{i, j}=n+1$ and $\sum_{i, j} j a_{i, j}=k$.

Formulas (31) and (22) imply that

$$
\frac{\partial^{n+k} \mu}{\partial t^{n} \partial s^{k}}(t, s)=0 \quad \text { if } t \leq 0
$$

and the function

$$
\frac{\partial^{n+k} \mu}{\partial t^{n} \partial s^{k}}(t, s):(0,+\infty) \times \mathbb{R} \rightarrow \mathbb{R}
$$

is a linear combination of the following finite set of functions:

(32) $\omega(t, s)$

$$
=\frac{\left(\varphi^{\prime}(t)\right)^{j_{1}} \cdots\left(\varphi^{(n+1)}(t)\right)^{j_{n+1}}\left(\beta^{\prime}\left(s-t^{-1}\right)\right)^{i_{1}} \cdots\left(\beta^{(n+k+1)}\left(s-t^{-1}\right)\right)^{i_{n+k+1}}}{\left(\varphi(t)+\beta\left(s-t^{-1}\right)\right)^{r} t^{l}},
$$

where $i_{q}, j_{q}, l \in \mathbb{Z}_{+}, r=\sum j_{q}+\sum i_{u} \in \mathbb{N}, l \leq 2 \sum i_{u}, \sum q j_{q} \leq n+1$ and $\sum u i_{u} \leq k$.

The inclusion $\mu \in \mathcal{B}$ will be proved if we show that

$$
|\omega(t, s)| \preccurlyeq(1+|s|)^{m}
$$

for any function $\omega$ defined in (32) $(m=m(\omega) \geq 0)$. From (32) and (21) it follows that

$$
\omega(t, s) \preccurlyeq 1 \quad \text { for } t \geq 1 / 2
$$

(the denominator in (32) is bounded from below by $\left.\varphi^{r}(1 / 2) 2^{-l}\right)$.

CASE 1: $\sum i_{u}>0$. According to $(21), \omega(t, s)=0$ if $s-t^{-1} \notin(0,1)$. Using (34) we obtain

$$
|\omega(t, s)| \preccurlyeq 1 \quad \text { for } s \leq 2 .
$$

Let $s>2$. If $1-s^{-2}<s-t^{-1}<1$, we have $\beta^{(i)}\left(s-t^{-1}\right) \preccurlyeq e^{-s^{4}} s^{6 i} \preccurlyeq \varphi(t) s^{6 i}$. If $0<s-t^{-1}<1-s^{-2}$, we have $\beta^{(i)}\left(s-t^{-1}\right) \preccurlyeq \beta\left(s-t^{-1}\right)\left(1-s+t^{-1}\right)^{-3 i} \preccurlyeq$ $\beta\left(s-t^{-1}\right) s^{6 i}$. Thus, if $s>2$ and $0<s-t^{-1}<1$ then $\beta^{(i)}\left(s-t^{-1}\right) \preccurlyeq(\varphi(t)+$ $\left.\beta\left(s-t^{-1}\right)\right) s^{6 i}$ and $\varphi^{(j)}(t) / \varphi(t) \preccurlyeq t^{-5 j} \preccurlyeq s^{5 j}$. Using (32) we deduce that if $s>2$ and $0<s-t^{-1}<1$ then $\omega(t, s) \preccurlyeq s^{m}$, where $m=l+5 \sum q j_{q}+6 \sum u i_{u}$. Since $\omega(t, s)=0$ if $s-t^{-1} \notin(0,1)$, this formula and (35) imply (33).

CASE 2: $\sum i_{u}=0$. In this case

$$
\omega(t, s)=\frac{\left(\varphi^{\prime}(t)\right)^{j_{1}} \cdots\left(\varphi^{(n+1)}(t)\right)^{j_{n+1}}}{\left(\varphi(t)+\beta\left(s-t^{-1}\right)\right)^{r}}
$$


where $j_{q} \in \mathbb{Z}_{+}$and $1 \leq r=\sum j_{q} \leq n+1$. If $s \leq 1 / 2$, or $s>1 / 2$ and $t \leq 1 /(s-1 / 2)$, then $\omega(t, s) \preccurlyeq 1$ (the denominator in (36) is bounded from below by $\left.2^{-r}\right)$. If $s>1 / 2$ and $t \geq 1 / s$ then $\varphi^{(j)}(t) / \varphi(t) \preccurlyeq t^{-5 j} \preccurlyeq s^{5 j}$ for any $j \in \mathbb{N}$. This inequality and (36) imply that if $s>1 / 2$ and $t \geq 1 / s$, then $\omega(t, s) \preccurlyeq s^{m}$, where $m=5 \sum q j_{q}$, which proves (33). Therefore $\mu \in \mathcal{B}$.

\section{References}

[1] K. Astala, On Peano's theorem in locally convex spaces, Studia Math. 73 (1982), $213-223$.

[2] V. I. Averbukh and O. G. Smolyanov, The various definitions of the derivative in linear topological spaces, Uspekhi Mat. Nauk 23 (1968), no. 4, 67-114 (in Russian); English transl.: Russian Math. Surveys 23 (1968), 67-113.

[3] C. Bessaga and A. Pełczyński, Selected Topics in Infinite-Dimensional Topology, PWN-Polish Sci. Publ., Warszawa, 1975.

[4] V. I. Bogachev, Deterministic and stochastic differential equations in infinite dimensional spaces, Acta Appl. Math. 40 (1995), 25-93.

[5] J. Bonet and P. Carreras, Barrelled Locally Convex Spaces, North-Holland, Amsterdam, 1987.

[6] A. N. Godunov, Linear differential equations in locally convex spaces, Vestnik Moskov. Univ. Ser. I Mat. Mekh. 29 (1974), no. 5, 31-39 (in Russian); English transl.: Moscow Univ. Math. Bull. 29 (1974), no. 5, 25-31.

[7] G. Herzog, On existence and uniqueness conditions for ordinary differential equations in Fréchet spaces, Studia Sci. Math. Hungar. 32 (1996), 367-375.

[8] -, One-sided estimates for ordinary differential equations in Fréchet spaces, Ricerche Mat. 46 (1997), 479-487.

[9] -, On ordinary linear differential equations in $\mathbb{C}^{J}$, Demonstratio Math. 28 (1995), 383-398.

[10] -, On linear time-dependent row-finite systems of differential equations, in: Travaux Math. 8, Sém. Math. Luxembourg, Centre Univ. Luxembourg, Luxembourg, 1996, $167-176$.

[11] -, On row-finite systems of differential equations in Banach spaces, Demonstratio Math. 31 (1998), 835-839.

[12] G. Herzog and R. Lemmert, Nonlinear fundamental systems for linear differential equations in Fréchet spaces, ibid. 33 (2000), 313-318.

[13] G. Köthe, Topological Vector Spaces I, Springer, New York, 1969; Topological Vector Spaces II, Springer, New York, 1979.

[14] K. Kuratowski, Topology, Vols. 1 and 2, Academic Press, New York, 1966 and 1968.

[15] R. Lemmert, On ordinary differential equations in locally convex spaces, Nonlinear Anal. 10 (1986), 1385-1390.

[16] A. K. Leonov and S. A. Shkarin, Integral completeness of locally convex spaces, Russian J. Math. Phys. 7 (2000), 402-412.

[17] S. G. Lobanov, Picard's theorem for ordinary differential equations in locally convex spaces, Izv. Ross. Akad. Nauk Ser. Mat. 56 (1992), 1217-1243 (in Russian); English transl.: Russian Acad. Sci. Izv. Math. 41 (1993), 465-487.

[18] -, An example of a non-normable Fréchet space in which every continuous linear operator has an exponential, Uspekhi Mat. Nauk 34 (1979), no. 4, 201-202 (in Russian); English transl.: Russian Math. Surveys 34 (1979), no. 4, 176-177. 
[19] S. G. Lobanov, Solvability of linear ordinary differential equations in locally convex spaces, Vestnik Moskov. Univ. Ser. Mat. Mekh. 1980, no. 2, 3-7 (in Russian); English transl.: Moscow Univ. Math. Bull. 35 (1980), no. 2, 1-5.

[20] S. G. Lobanov and O. G. Smolyanov, Ordinary differential equations in locally convex spaces, Uspekhi Mat. Nauk 49 (1994), 93-168 (in Russian); English transl.: Russian Math. Surveys 49 (1994), 97-175.

[21] R. Meise and D. Vogt, Introduction to Functional Analysis, Oxford Univ. Press, New York, 1997.

[22] E. Michael, A selection theorem, Proc. Amer. Math. Soc. 17 (1966), 1404-1406.

[23] V. M. Millionshchikov, On the theory of differential equations in locally convex spaces, Mat. Sb. 57 (1962), 385-406 (in Russian); English transl.: Math. USSR-Sb. 57 (1962), 361-379.

[24] J. Oxtoby, Measure and Category. A Survey of Analogies Between Topological and Measure Spaces, Springer, New York, 1971.

[25] M. Reed and B. Simon, Methods of Modern Mathematical Physics. 1. Functional Analysis, Academic Press, New York, 1970.

[26] L. Schwartz, Analyse Mathématique, Hermann, Paris, 1967.

[27] S. A. Shkarin, On stratifiable locally convex spaces, Russian J. Math. Phys. 6 (1999), $435-460$.

[28] -, Whitney's type theorems for infinite dimensional spaces, Infin. Dimens. Anal. Quantum Probab. Relat. Top. 3 (2000), 141-160.

[29] - Differential equations and smooth functions in locally convex spaces, Ph.D. thesis, Moscow, 1991 (in Russian).

[30] - Peano's theorem in infinite-dimensional Fréchet spaces is invalid, Funktsional. Anal. i Prilozhen. 27 (1993), no. 2, 90-92 (in Russian); English transl.: Funct. Anal. Appl. 27 (1993), no. 2, 149-151.

[31] - Peano's theorem is invalid in infinite-dimensional $F^{\prime}$-spaces, Mat. Zametki 62 (1997), 128-137 (in Russian); English transl.: Math. Notes 62 (1997), 108-115.

[32] - Some results on solvability of ordinary linear differential equations in locally convex spaces, Mat. Sb. 181 (1990), 1183-1195 (in Russian); English transl.: Math. USSR-Sb. 71 (1992), 29-40.

[33] - On solvability of linear differential equations in $\mathbb{R}^{\mathbb{N}}$, Demonstratio Math. 38 (2005), 85-99.

[34] S. Szufla, On the equation $x^{\prime}=f(t, x)$ in locally convex spaces, Math. Nachr. 118 (1984), 179-185.

[35] M. Valdivia, Topics in Locally Convex Spaces, North-Holland, Amsterdam, 1982.

Mathematics Department

King's College London, Strand

London WC2R 2LS, UK

E-mail: shkarin@mth.kcl.ac.uk

shkarin@math.uni-wuppertal.de

Received July 22, 2002

Revised version October 6, 2005 